\title{
PARTIAL ORDERS ON CONJUGACY CLASSES IN THE WEYL GROUP AND ON UNIPOTENT CONJUGACY CLASSES
}

\author{
JEFFREY ADAMS, XUHUA HE, AND SIAN NIE
}

\begin{abstract}
Let $G$ be a reductive group over an algebraically closed field and let $W$ be its Weyl group. In a series of papers, Lusztig introduced a map from the set $[W]$ of conjugacy classes of $W$ to the set $\left[G_{u}\right]$ of unipotent classes of $G$. This map, when restricted to the set of elliptic conjugacy classes $\left[W_{e}\right]$ of $W$, is injective. In this paper, we show that Lusztig's map $\left[W_{e}\right] \rightarrow\left[G_{u}\right]$ is order-reversing, with respect to the natural partial order on $\left[W_{e}\right]$ arising from combinatorics and the natural partial order on $\left[G_{u}\right]$ arising from geometry.
\end{abstract}

\section{Contents}

Introduction 1

1. Main result 4

2. Unipotent conjugacy classes of classical groups 6

3. Lusztig's map for classical groups 9

4. Examples 13

5. Proof of the Main Theorem for classical groups 15

6. Exceptional Groups 20

References 24

\section{INTRODUCTION}

0.1. Lusztig's map. Let $G$ be a connected reductive group over an algebraically closed field $\mathbb{F}$ and let $W$ be the Weyl group of $G$. Let $\left[G_{u}\right]$ be the set of unipotent conjugacy classes in $G$ and $[W]$ be the set of conjugacy classes of $W$. Lusztig defined a surjective map $\Phi:[W] \rightarrow\left[G_{u}\right]$ ([Lu11, Theorem 0.4]). This construction was generalized to twisted conjugacy classes in [Lu12b].

Roughly speaking, the map $\Phi$ is constructed as follows. Let $\mathcal{C} \in[W]$ and $w \in \mathcal{C}_{\text {min }}$ be a minimal length element of $\mathcal{C}$. We look at the intersection of the Bruhat double coset $B w B$ with unipotent conjugacy classes and we select the minimal unipotent class which gives a nonempty intersection. It is pointed out in $[\mathrm{Lu} 11, \S 0.1]$ that "the fact that the procedure actually works is miraculous".

In this paper we are concerned with the set of elliptic conjugacy classes $\left[W_{e}\right] \subset$ $[W]$. The restriction of $\Phi$ to $\left[W_{e}\right]$ is injective, and the image contains all distinguished unipotent conjugacy classes ([Lu11, Proposition 0.6]).

Date: April 6, 2020.

2010 Mathematics Subject Classification. Primary: 20G07, Secondary: 06A07, 20F55, 20E45.

Key words and phrases. Reductive groups, Weyl groups, conjugacy classes, partial orders. 
The set $\left[G_{u}\right]$ has the natural partial ordering by closure relations, denote $\preceq_{u}$. On the other hand the second-named author [He07] (see also [He16a, §1.10.3]) introduced a partial order on $\left[W_{e}\right]$, induced from the Bruhat order on minimal length elements of the elliptic conjugacy classes of $W$, which we denote $\preceq_{W}$.

It is a natural question to consider how Lusztig's map behaves with respect to these partial orders. Dudas, Michel and the second-named author [DHM13] conjectured that $\Phi$ gives an order-reversing bijection from $\left[W_{e}\right]$ to $\Phi\left(\left[W_{e}\right]\right) \subset\left[G_{u}\right]$. Michel [DHM13] verified the exceptional groups by computer.

In [DM15, Conjecture 3.7], Dudas and Malle conjectured that a similar result holds for twisted type $A$. In [DM15, Proposition $3.11 \& 3.14]$ they verified the conjecture for some special family of twisted elliptic conjugacy classes of type $A$. It is also verified in [DM15] by computer that the conjecture holds for twisted $A_{n}$ with $n \leqslant 10$. The compatibility of the partial orders is used in [DM15, §5] to study the decomposition numbers of the unipotent $l$-blocks of finite unitary groups.

Our first main result is that $\Phi$ is order reversing in the following sense.

Theorem 0.1. Let $\mathcal{C}, \mathcal{C}^{\prime} \in\left[W_{e}\right]$. Then $\mathcal{C} \preceq_{W} \mathcal{C}^{\prime}$ if and only if $\Phi\left(\mathcal{C}^{\prime}\right) \preceq_{u} \Phi(\mathcal{C})$.

This holds also in the twisted setting.

0.2. A similar phenomenon for affine Weyl groups. Before we discuss the strategy towards Theorem 0.1, we make a short digression and discuss a similar phenomenon. For simplicity, we only discuss split groups here but the result holds in general.

Let $G$ be a connected reductive group. The Frobenius morphism $\sigma$ of $\overline{\mathbb{F}_{q}}$ over $\mathbb{F}_{q}$ induces a Frobenius morphism $\sigma$ on $G\left(\overline{\mathbb{F}_{q}}((t))\right)$. Let $B(G)$ be the set of $\sigma$-twisted conjugacy classes on $G\left(\overline{\mathbb{F}_{q}}((t))\right)$. Let $\tilde{W}$ be the Iwahori-Weyl group and let $[\tilde{W}]$ be the set of conjugacy classes of $\tilde{W}$. Inside $[\tilde{W}]$, there is a special subset, the set of straight conjugacy classes, which we denote by $\left[\tilde{W}_{s t r}\right]$. It is proved in [He14] that there is a natural bijection $\tilde{\Phi}:\left[\tilde{W}_{s t r}\right] \rightarrow B(G)$, which is induced from any lifting $\tilde{W} \rightarrow G\left(\overline{\mathbb{F}_{q}}((t))\right)$.

The closure relation gives the partial order on $B(G)$. There is also a partial order on $\left[\tilde{W}_{s t r}\right]$ induced from the Bruhat order on $\tilde{W}$, similar to the definition of the partial order $\preceq_{W}$ on $\left[W_{e}\right]$ we discussed earlier. It is proved in [He16b, Theorem $\mathrm{B}]$ that these two partial orders coincide via the bijection $\tilde{\Phi}:\left[\tilde{W}_{s t r}\right] \rightarrow B(G)$.

The proof uses the reduction method of Deligne and Lusztig [DL76], some remarkable combinatorial properties on the straight conjugacy classes [HN14] and a deep result in arithmetic geometry, the purity theorem for the Newton stratification associated with $F$-crystal obtained by de Jong-Oort [JO00], Hartl-Viehmann [HV11], Viehmann [Vi13] and Hamacher [Ha15].

0.3. Difference between the finite and affine cases. Although in both finite and affine cases, we compare the partial orders arising from combinatorics and from geometry, there are some essential differences between the two cases we discussed above.

First, in the affine case, we do not consider the conjugation action on the loop groups, but the Frobenius-twisted conjugacy classes instead. Although the study of the Frobenius-twisted conjugacy classes are quite involved, it is, in some sense, simpler than the unipotent conjugacy classes. Second, the construction of the 
map from conjugacy classes of Weyl groups to the (twisted) conjugacy classes of reductive groups in the finite and affine case are quite different. In the affine case, the map is induced from any lifting $\tilde{W} \rightarrow G\left(\overline{\mathbb{F}_{q}}((t))\right)$, while in the finite case the construction is rather a miracle. Finally, in the affine case, the map preserves the partial orders, while in the finite case, as we show in this paper, the map reverses the partial orders.

0.4. The strategy. Now we discuss the strategy towards the proof of Theorem 0.1. For exceptional groups, we verify the statement by computer in Section 6. For classical groups, the elliptic conjugacy classes are parametrized by certain partitions. For such partition $\alpha$, we denote by $\mathcal{C}_{\alpha}$ the corresponding elliptic conjugacy class. We show that

$$
\Phi\left(\mathcal{C}_{\alpha}\right) \preceq_{u} \Phi\left(\mathcal{C}_{\beta}\right) \Leftrightarrow \alpha \leqslant \beta \Leftrightarrow \mathcal{C}_{\beta} \preceq_{W} \mathcal{C}_{\alpha}
$$

Note that the unipotent classes in the classical groups are associated to certain partitions. However, the partitions associated to elliptic conjugacy classes and the unipotent classes of the same classical group, are usually partitions of different integers. For example, in type $B_{n}$, the elliptic conjugacy classes correspond to partitions of $n$ while the unipotent conjugacy classes correspond to certain partitions of $2 n+1$. The map from partitions of $n$ to partitions of $2 n+1$ induced from Lusztig's map in characteristic 0 (and any characteristic $\neq 2$ ) are rather complicated; however, in characteristic 2 the map is rather simple and is essentially the map $\alpha \mapsto(2 \alpha, 1)$. We then use the Lusztig-Spaltenstein map from the set of unipotent classes in characteristic 0 to the set of unipotent classes in characteristic 2 to reduce the statement

$$
\Phi\left(\mathcal{C}_{\alpha}\right) \preceq_{u} \Phi\left(\mathcal{C}_{\beta}\right) \Leftrightarrow \alpha \leqslant \beta
$$

in characteristic 0 (and any characteristic $\neq 2$ ) to the statement $(*)$ in characteristic 2, which is obvious since the map $\alpha \mapsto(2 \alpha, 1)$. For other classical groups, the statement $\Phi\left(\mathcal{C}_{\alpha}\right) \preceq_{u} \Phi\left(\mathcal{C}_{\beta}\right) \Leftrightarrow \alpha \leqslant \beta$ is verified in the same way. This is done in section 3 .

We then verify the statement

$$
\alpha \leqslant \beta \Leftrightarrow \mathcal{C}_{\beta} \preceq_{W} \mathcal{C}_{\alpha}
$$

for classical groups.

By definition, $\mathcal{C}_{\beta} \preceq_{W} \mathcal{C}_{\alpha}$ if and only if there exist minimal length elements $w_{\beta} \in \mathcal{C}_{\beta}$ and $w_{\alpha} \in \mathcal{C}_{\alpha}$ such that $w_{\beta} \leqslant w_{\alpha}$. In [He07], the second-named author constructed explicit minimal length representatives for any elliptic conjugacy class of Weyl groups of classical type. If $\alpha \leqslant \beta$, then we have the desired relation between those minimal length representatives with respect to the Bruhat order. This proves the $\Rightarrow$ direction of the statement $(* *)$ for classical groups.

The $\Leftarrow$ direction of the statement $(* *)$ is more involved. The difficulty is that there are many minimal length elements in a given elliptic conjugacy class and the number is unbounded as the rank of the group becomes larger. To overcome the difficulty, we use the explicit description of the Bruhat order for the Weyl groups of classical type (see [BB05]). For example, for any elements $w$ in the Weyl group of type $B_{n}$ and $-n \leqslant i, j \leqslant n$, we may associate a nonnegative integer $w[i, j]$. Then $w \leqslant w^{\prime}$ if and only if $w[i, j] \leqslant w^{\prime}[i, j]$ for all $-n \leqslant i, j \leqslant n$. So there are $4 n^{2}$ inequalities to check. Fortunately, to study $\mathcal{C}_{\beta} \preceq_{W} \mathcal{C}_{\alpha}$, one only needs to 
investigate $l$ inequalities among all the $4 n^{2}$ inequalities, where $l$ is the number of parts in the partition $\beta$. And these $l$ inequalities imply that $\alpha \leqslant \beta$. This is done in section 5 .

Acknowledgements: The idea that the partial orders on the conjugacy classes of Weyl groups and the unipotent classes of algebraic groups might be related was initiated in the private conversation of the second-named author with Olivier Dudas and Jean Michel. The explicit description of the Bruhat order for classical groups was pointed out to us by Thomas Lam. We also thank George Lusztig and Zhiwei Yun for helpful discussions.

\section{MAIN RESUlT}

1.1. Preliminary. Let $G$ be an affine algebraic group over an algebraically closed field $\mathbb{F}$ of characteristic $p \geqslant 0$ such that the identity component $G^{0}$ of $G$ is reductive. Let $T$ be a maximal torus of $G^{0}$ and $B \supset T$ be a Borel subgroup of $G^{0}$. Let $W^{0}=N_{G^{0}}(T) / T$ be the Weyl group of $G^{0}$ and let $W=N_{G}(T) / T$ be the (extended) Weyl group of $G$. The length function $\ell$ on $W^{0}$ extends in a unique way to a length function on $W$, which we still denote by $\ell$. Let $S \subset W^{0}$ be a set of simple reflections.

Let $D$ be a connected component of $G$ and $W^{D}=\left(N_{G}(T) \cap D\right) / T$ be a left/right $W^{0}$-coset of $W$. By [Lu03, Section 1.4], $W^{D}$ contains a unique element $\epsilon_{D}$ of length 0 . The conjugation action of $\epsilon_{D}$ on $W$ is a length-preserving automorphism. Let $[W]$ be the set of $W^{0}$-conjugacy classes of $W$ and $\left[W^{D}\right]$ be the set of $W^{0}$-conjugacy classes of $W$ that intersect $D$. An element $w \in W^{D}$ (or its $W^{0}$-conjugacy class $C$ in $W$ ) is said to be elliptic if for any $J \varsubsetneqq S$ with $\operatorname{Ad}\left(\epsilon_{D}\right)(J)=J$, we have $C \cap W_{J} \epsilon_{D}=\emptyset$. Let $\left[W_{e}^{D}\right]$ the set of elliptic $W^{0}$-conjugacy classes of $W$ that intersect $W^{D}$.

From now on, we assume that $D$ contains a unipotent element of $G$. Let $\left[D_{u}\right]$ be the set of $G^{0}$-conjugacy classes of $D$ which are unipotent. In [Lu11] and [Lu12b], Lusztig introduced a map $\Phi:\left[W^{D}\right] \rightarrow\left[D_{u}\right]$. It is proved in loc. cit. that

- The map $\Phi:\left[W^{D}\right] \rightarrow\left[D_{u}\right]$ is surjective.

- The restriction to elliptic conjugacy classes $\Phi_{e}:\left[W_{e}^{D}\right] \rightarrow\left[D_{u}\right]$ is injective.

1.2. Partial orders. We define a partial order on unipotent classes by the closure relations as usual: $\mathcal{C} \preceq_{u} \mathcal{C}^{\prime}$ if $\mathcal{C} \subset \overline{\mathcal{C}^{\prime}}$.

Now we recall the partial order on $\left[W_{e}^{D}\right]$ introduced in $[$ He07, $\S 4.7]$. Let $\mathcal{C}, \mathcal{C}^{\prime} \in$ $\left[W_{e}^{D}\right]$. We denote by $\mathcal{C}_{\text {min }}$ (respectively $\mathcal{C}_{\text {min }}^{\prime}$ ) the set of minimal length elements in $\mathcal{C}$ (respectively $\mathcal{C}^{\prime}$ ). Then the following conditions are equivalent:

(1) For some $w \in \mathcal{C}_{\text {min }}$, there exists $w^{\prime} \in \mathcal{C}_{\text {min }}^{\prime}$ such that $w^{\prime} \leqslant w$;

(2) For any $w \in \mathcal{C}_{\min }$, there exists $w^{\prime} \in \mathcal{C}_{\text {min }}^{\prime}$ such that $w^{\prime} \leqslant w$.

If these conditions are satisfied, then we write $\mathcal{C}^{\prime} \preceq_{W} \mathcal{C}$. By the equivalence of the conditions (1) and (2) above, the relation $\preceq_{W}$ is transitive. This gives a natural partial order on the set $\left[W_{e}^{D}\right]$.

By [He07, Corollary 4.5], $\mathcal{C}_{\text {min }}^{\prime}$ is the set of minimal elements in $\mathcal{C}^{\prime}$ with respect to the Bruhat order of $W$. Thus the conditions (1) and (2) above are also equivalent to the following conditions:

(3) For some $w \in \mathcal{C}_{\text {min }}$, there exists $w^{\prime} \in \mathcal{C}^{\prime}$ such that $w^{\prime} \leqslant w$; 
(4) For any $w \in \mathcal{C}_{\min }$, there exists $w^{\prime} \in \mathcal{C}^{\prime}$ such that $w^{\prime} \leqslant w$.

The condition (3) will be used to study the partial order on $\left[W_{e}^{D}\right]$ for exceptional groups.

Now we state the main theorem of the paper.

Theorem 1.1. The map $\Phi_{e}:\left[W_{e}^{D}\right] \rightarrow\left[D_{u}\right]^{\text {op }}$ gives a bijection from the poset $\left[W_{e}^{D}\right]$ to its image. Here $\left[D_{u}\right]^{\text {op }}$ is the same as $\left[D_{u}\right]$ as a set, but with reversed partial order.

In other words let $\mathcal{C}, \mathcal{C}^{\prime} \in\left[W_{e}^{D}\right]$. Then $\mathcal{C}^{\prime} \preceq_{W} \mathcal{C}$ if and only if $\Phi(\mathcal{C}) \preceq_{u} \Phi\left(\mathcal{C}^{\prime}\right)$.

1.3. Reduction to almost simple groups. In this subsection, we show that to prove Theorem 1.1, it suffices to consider the case where $G^{0}$ is simple. The reduction procedure is the similar to [Lu12b, §1.5-1.11].

First, we may replace $G$ by the subgroup generated by $D$. Next, let $G^{\prime}=$ $G / Z\left(G^{0}\right)$ and $\pi: G \rightarrow G^{\prime}$ be projection map. As the Weyl groups of $G$ and $G^{\prime}$ are naturally identical, Theorem 1.1 holds for $(G, D)$ if and only if it holds for $\left(G^{\prime}, D^{\prime}\right)$, where $D^{\prime}=\pi(D)$.

Now we may assume that $G^{0}$ is semisimple and simply connected. We write $G^{0}$ as $G^{0}=G_{1} \times \ldots \times G_{k}$, where each $G_{i} \neq\{1\}$ is a minimal closed connected normal subgroup of $G$. For any $i$, let $G_{i}^{\prime}=G /\left(G_{1} \times \ldots \times \hat{G}_{i} \times \ldots \times G_{k}\right)$ and $D_{i}$ be the image of $D$ in $G_{i}^{\prime}$. Let $G^{\prime}=G_{1}^{\prime} \times \ldots \times G_{k}^{\prime}$. We may then identify $G$ with a closed subgroup of $G^{\prime}$ with the same identity component. Under this identification, $D$ becomes $D_{1} \times \ldots \times D_{k}$. Let $W^{\prime}$ be the extended Weyl group of $G^{\prime}$ and $W_{i}^{\prime}$ be the extended Weyl group of $G_{i}^{\prime}$. Then $W^{\prime}=W_{1}^{\prime} \times \ldots \times W_{k}^{\prime}$ and we may identify $W^{D}$ with $\left(W_{1}^{\prime}\right)^{D_{1}} \times \ldots \times\left(W_{k}^{\prime}\right)^{D_{k}}$. Under this identification,

$$
\left[W_{e}^{D}\right]=\left[\left(W_{1}^{\prime}\right)_{e}^{D_{1}}\right] \times \ldots \times\left[\left(W_{k}^{\prime}\right)_{e}^{D_{k}}\right]
$$

and $\preceq_{W}$ on $\left[W_{e}^{D}\right]$ coincides with $\preceq_{W, 1} \times \ldots \times \preceq_{W, k}$ on $\left[\left(W_{1}^{\prime}\right)_{e}^{D_{1}}\right] \times \ldots \times\left[\left(W_{k}^{\prime}\right)_{e}^{D_{k}}\right]$. By $[$ Lu12b, $\S 1.8], \Phi_{e}$ on $\left[W_{e}^{D}\right]$ coincides with $\Phi_{e, 1} \times \ldots \times \Phi_{e, k}$ on $\left[\left(W_{1}^{\prime}\right)_{e}^{D_{1}}\right] \times \ldots \times$ $\left[\left(W_{k}^{\prime}\right)_{e}^{D_{k}}\right]$. Thus if Theorem 1.1 holds for each $\left(G_{i}^{\prime}, D_{i}\right)$, then it holds for $(G, D)$.

Now we may assume that $G^{0}$ is semisimple, simply connected and that $G$ has no nontrivial closed connected normal subgroups. By [Lu12b, §1.9], $G^{0}=H_{1} \times \ldots \times$ $H_{m}$, where $H_{i}$ are connected, simply connected, almost simple, closed subgroups of $G^{0}$ and there exists a $c \in D$ such that $H_{i}=c^{i} H_{1} c^{-i}$ for $0 \leqslant i \leqslant m-1$ and $c^{m} H_{1} c^{-m}=H_{1}$. Let $G^{\prime}$ be the subgroup of $G$ generated by $H_{1}$ and $c^{m}$ and $D^{\prime}=c^{m} H_{0}$ be a connected component of $G^{\prime}$. By [Lu12b, $\left.\S 1.9\right]$, we may identify $\left[D_{u}\right]$ with $\left[D_{u}^{\prime}\right]$ and $\left[W^{D}\right]$ with $\left[\left(W^{\prime}\right)^{D^{\prime}}\right]$ and under this identification, we have the following commutative diagram

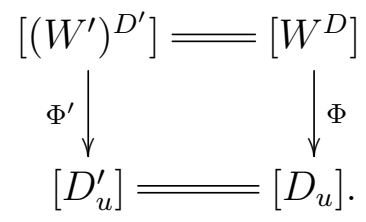

Thus Theorem 1.1 holds for $(G, D)$ if and only if it holds for $\left(G^{\prime}, D^{\prime}\right)$.

Therefore, to prove Theorem 1.1, it suffices to consider the cases where $G^{0}$ is almost simple. 


\section{UNIPOTENT CONJUGACY CLASSES OF CLASSICAL GROUPS}

In this section, we recollect some facts on the unipotent conjugacy classes of classical groups, over an algebraically closed field $\mathbb{F}$ of any characteristic. We follow [Sp82, Section I.2].

Suppose $V$ is a finite-dimensional vector space over $\mathbb{F}$. We define a disconnected group containing $\mathrm{GL}(V)(\operatorname{dim}(V) \geqslant 3)$ as in [Sp82, Section I.2.7]. Define $\mathrm{GL}^{\dagger}(V)=G_{0} \cup G_{1}$ where $G_{0}=G L(V)$ and $G_{1}$ is the set of non-singular bilinear forms $\phi: V \times V \rightarrow \mathbb{F}$. We define the product structure on $G$ as follows. The product on $G_{0}$ is the usual one. If $g \in G_{0}, \phi \in G_{1}$ then $(g \phi)(v, w)=\phi\left(g^{-1} v, w\right)$ and $(\phi g)(v, w)=\phi(v, g w)$. If $\phi, \psi \in G_{1}$ then $\phi \psi$ is the unique element of $G_{0}$ satisfying $\phi((\phi \psi) v, w)=\psi(w, v)$.

It is easy to see $\mathrm{GL}^{\dagger}(V)$ is a group and $\mathrm{GL}^{\dagger}(V) / \mathrm{GL}(V) \simeq \mathbb{Z} / 2 \mathbb{Z}$. Also $\phi$ is symmetric if and only if $\phi^{2}=1$, so there is a unique $\operatorname{GL}(V)$-conjugacy class of such elements. Choose a basis of $V$ and identify $\operatorname{GL}(V)$ with $\operatorname{GL}(n)$, and set $\delta(v, w)=v \cdot w$. Then $\delta^{2}=1$ and $\delta g \delta={ }^{t} g^{-1}$ for $g \in \operatorname{GL}(V)$.

By a classical group we mean one of the groups $\mathrm{GL}(n), \mathrm{Sp}(2 n), \mathrm{SO}(n), \mathrm{O}(n)$ or $\mathrm{GL}^{\dagger}(n)$. The groups $\mathrm{GL}(n)$ and $\mathrm{Sp}(2 n)$ are connected. The identity component of $\mathrm{O}(n)$ is $\mathrm{SO}(n)$, and $\mathrm{O}(n) / \mathrm{SO}(n)$ is trivial if $p=2$, and has order 2 otherwise.

Let $\mathcal{P}(n)$ be the set of partitions of $n$. We write a partition of $n$ as $\alpha=$ $\left(\alpha_{1}, \ldots, \alpha_{\ell}\right)$ with $\alpha_{1} \geqslant \ldots \geqslant \alpha_{\ell} \geqslant 0$ and $\sum \alpha_{i}=n$. On occasion we do allow some $\alpha_{i}$ to be 0 , i.e., we regard $\left(\alpha_{1}, \ldots, \alpha_{\ell}\right)$ and $\left(\alpha_{1}, \ldots, \alpha_{\ell}, 0\right)$ to be the same partition. We define the standard partial order on partitions of the same integer $n: \alpha \leqslant \beta$ if for all $k, \sum_{i=1}^{k} \alpha_{i}=\sum_{i=1}^{k} \beta_{k}$.

We identify partitions with Young diagrams, and define the transpose partition as usual. If $\alpha=\left(\alpha_{1}, \ldots, \alpha_{\ell}\right)$ is a partition we let $\alpha^{*}=\left(\alpha_{1}^{*}, \ldots, \alpha_{m}^{*}\right)$ be the transpose partition. In particular $\alpha_{1}^{*}$ is the number of rows of $\alpha$.

Given a partition $\alpha$ define the multiplicity function $m_{\alpha}: \mathbb{Z}_{\geqslant 0} \rightarrow \mathbb{Z}_{\geqslant 0}$ as usual: $m_{\alpha}(k)=\left|\left\{j \mid \alpha_{j}=k\right\}\right|$. In particular $m_{\alpha}(k)=0$ for $k=0$ or $k>\alpha_{1}$. For $\kappa= \pm 1$ let

$$
\mathcal{P}_{\kappa}(n)=\left\{\alpha \in \mathcal{P}(n) \mid m_{\alpha}(i) \text { is even if }(-1)^{i}=\kappa\right\}
$$

Let $\mathcal{P}(n)_{0}$ be the partitions of $n$ with an even number of parts, i.e. with $\alpha_{1}^{*}$ even and $\mathcal{P}(n)_{1}$ be the partitions of $n$ with an odd number of parts, i.e. with $\alpha_{1}^{*}$ odd. Let $\mathcal{P}(n)^{\text {odd }}$ be the partitions consisting only of odd parts.

2.1. Unipotent classes in good characteristic. If $G=\mathrm{GL}^{\dagger}(n), \operatorname{Sp}(2 n)$ or $\mathrm{O}(n)$ then the characteristic $p=2$ is said to be bad (for $G$ ). Otherwise, including all groups in characteristic 0 , the characteristic is said to be good.

Suppose the characteristic of $\mathbb{F}$ is good. Then every unipotent element of $G$ is contained in $G^{0}$, and the unipotent classes are parametrized as follows.

(1) $\mathrm{GL}(n)$ or $\mathrm{GL}^{\dagger}(n): \mathcal{P}(n)$;

(2) $\mathrm{O}(2 n+1): \mathcal{P}_{1}(2 n+1)$;

(3) $\operatorname{Sp}(2 n): \mathcal{P}_{-1}(2 n)$;

(4) $\mathrm{O}(2 n): \mathcal{P}_{1}(2 n)$

We also consider the unipotent conjugacy classes of $\mathrm{SO}(n)$. If $n$ is odd the unipotent conjugacy class of $\mathrm{SO}(n)$ and $O(n)$ are in bijection, and the same holds for $\mathrm{SO}(2 n)$ if $n$ is odd. If $n$ is even the unipotent $\mathrm{SO}(2 n)$-conjugacy classes in 
$\mathrm{SO}(2 n)$ are parametrized by $\mathcal{P}_{1}(2 n)$, except that every partition with only even parts corresponds to two classes; there are $p(n / 2)$ of these classes where $p$ is the partition function. The $\mathrm{O}(2 n)$ orbits which split into two $\mathrm{SO}(2 n)$ orbits do not arise in the image of Lusztig's map applied to elliptic conjugacy classes, so we do not need to distinguish these two classes.

We write $\mathcal{U}_{\alpha}$ for the unipotent class parametrized by a partition $\alpha$. Then the partial order on unipotent conjugacy classes is given by the partial order on partitions: $\mathcal{U}_{\alpha} \preceq_{u} \mathcal{U}_{\beta}$ if and only if $\alpha \leqslant \beta$.

2.2. Unipotent conjugacy classes in bad characteristic. Suppose $G$ is a classical group and the characteristic $p$ of $\mathbb{F}$ is bad, in particular $p=2$. There is a bijective algebraic group homomorphism from $\operatorname{Sp}(2 n)$ to $S O(2 n+1)$ (although the inverse is not algebraic), which induces a bijection of unipotent classes. Also $S O(2 n+1)=O(2 n+1)$ so we do not need to consider these groups.

2.2.1. The cases $G=S p(2 n)$ and $O(2 n)$. Consider a set $\{\omega, 0,1\}$ where $\omega$ is a formal element, satisfying $\omega<0<1$. For $n \geqslant 1$ define $\widetilde{\mathcal{P}_{-1}}(n)$ to be the set of pairs $(\alpha, \epsilon)$, where

(1) $\alpha \in \mathcal{P}_{-1}(2 n)$ (i.e. odd rows have even multiplicity);

(2) $\epsilon: \mathbb{Z}_{\geqslant 0} \rightarrow\{\omega, 0,1\}$.

The function $\epsilon$ is required to satisfy, for all $i \geqslant 0$ :

$$
\epsilon(i)= \begin{cases}1, & \text { if } i=0, G=S p(n) \\ 0, & \text { if } i=0, G=O(n) \\ \omega, & \text { if } i \text { odd; } \\ \omega, & \text { if } i>0, m_{\alpha}(i)=0 \\ 1, & \text { if } i>0 \text { even, } m_{\alpha}(i) \text { odd; } \\ 0 \text { or } 1, & \text { if } i>0 \text { even, } m_{\alpha}(i)>0 \text { even. }\end{cases}
$$

Note that $\widetilde{\mathcal{P}_{-1}}(n)$ is empty if $n$ is odd, and $\epsilon(i)$ is determined by $\alpha$ except for even rows of even multiplicity.

Proposition 2.1. If $p=2$ the unipotent conjugacy classes in $S p(2 n)$ or $O(2 n)$ are in bijection with $\widetilde{\mathcal{P}_{-1}}(2 n)$.

We write $\mathcal{U}_{\alpha, \epsilon}$ for the unipotent class parametrized by $(\alpha, \epsilon)$.

The map from unipotent classes to $\mathcal{P}_{-1}(n)$ is defined as follows. First assume $G=S p(2 n)$, and let $\langle$,$\rangle be the symplectic form defining G$. We embed $\phi$ : $\operatorname{Sp}(2 n) \rightarrow G^{*}=G L(2 n)$ as usual. If $g \in G$ is unipotent then $\phi(g) \in G^{*}$ is unipotent, and so corresponds to a partition $\alpha$ of $2 n$; it is easy to see $\alpha \in \mathcal{P}_{-1}(2 n)$.

Suppose $i>0$ is even and $m_{\alpha}(i)>0$. Set $\epsilon(i)=0$ if $\left\langle(g-1)^{i-1} v, v\right\rangle=0$ for all $v \in \operatorname{ker}(g-1)^{i}$, and $\epsilon(i)=1$ otherwise. Together with the conditions above this defines $\epsilon$ uniquely. 
Next, if $G=O(2 n)$ we note that every unipotent conjugacy class in $S p(2 n)$ intersects $O(2 n)$ in a unique conjugacy class, and this defines a bijection between unipotent conjugacy classes in $S p(2 n)$ and $O(2 n)$.

Write $\mathcal{U}_{\alpha, \epsilon}$ for the unipotent conjugacy class associated to $(\alpha, \epsilon) \in \widetilde{\mathcal{P}_{-1}}(2 n)$.

In the case of $G=O(2 n)$ we need to distinguish between those $G$-conjugacy classes contained in $\mathrm{SO}(2 n)$ and those which are not.

Define $\widetilde{\mathcal{P}_{-1}}(2 n)_{0} \subset \widetilde{\mathcal{P}_{-1}}(2 n)$ to be the pairs $(\alpha, \epsilon)$ such that $\alpha_{1}^{*}$ is even, and set $\widetilde{\mathcal{P}_{-1}}(2 n)_{1}=\widetilde{\mathcal{P}_{-1}}(2 n) \backslash \widetilde{\mathcal{P}_{-1}}(2 n)_{0}$.

Lemma 2.2. Suppose $(\alpha, \epsilon) \in \widetilde{\mathcal{P}_{-1}}(2 n)$. Then $\mathcal{U}_{\alpha, \epsilon} \subset \mathrm{SO}(2 n)$ if and only if $(\alpha, \epsilon) \in \widetilde{\mathcal{P}_{-1}}(2 n)_{0}$.

Thus $\widetilde{\mathcal{P}_{-1}}(2 n)_{0}$ (respectively $\left.\widetilde{\mathcal{P}_{-1}}(2 n)_{1}\right)$ is in bijection with the unipotent $\mathrm{O}(2 n)$ conjugacy classes in $\mathrm{SO}(2 n)$ (respectively $\mathrm{O}(2 n) \backslash \mathrm{SO}(2 n)$ ).

Finally we consider unipotent $\mathrm{SO}(2 n)$-conjugacy classes. If $(\alpha, \epsilon) \in \widetilde{\mathcal{P}_{-1}}(2 n)_{0}$ then $\mathcal{U}_{\alpha, \epsilon} \subset \mathrm{SO}(2 n)$ is the union of two $\mathrm{SO}(2 n)$-conjugacy classes if for all $i, \alpha_{i}$ and $m_{\alpha}(i)$ are even and $\epsilon(i)=0$. Otherwise $\mathcal{U}_{\alpha, \epsilon} \subset \mathrm{SO}(2 n)$ is a single $\mathrm{SO}(2 n)$ conjugacy class. Again the $\mathrm{O}(2 n)$ orbits which split into two $\mathrm{SO}(2 n)$ orbits do not arise in the image of Lusztig's map applied to elliptic elements, so we do not need to distinguish these two classes.

2.2.2. The case $G=\mathrm{GL}^{\dagger}(n)$. Recall every characteristic for $\mathrm{GL}(n)$ is good, and the unipotent classes for $\operatorname{GL}(n)$ are parametrized by partitions of $n$. Now we consider $\mathrm{GL}^{\dagger}(n)(n \geqslant 3)$. Recall we write $\mathrm{GL}^{\dagger}(n)=G_{0} \cup G_{1}$ where $G_{0}=\operatorname{GL}(n)$ and $G_{1}$ is the set of non-singular bilinear forms.

Lemma 2.3. The unipotent conjugacy classes of $\mathrm{GL}^{\dagger}(n)$ which are contained in $\mathrm{GL}(n)$ are in bijection with the unipotent conjugacy classes of $\mathrm{GL}(n)$.

In other words if $\mathcal{U} \subset \mathrm{GL}(n)$ is a unipotent conjugacy class for $\mathrm{GL}^{\dagger}(n)$ then it is a single $\mathrm{GL}(n)$-orbit.

So consider the unipotent conjugacy classes of $\mathrm{GL}^{\dagger}(n)$ in $\mathrm{GL}^{\dagger}(n) \backslash \mathrm{GL}(n)$.

Define $\widetilde{\mathcal{P}_{1}}(n)$ to be the set of pairs $(\alpha, \epsilon)$, where

(1) $\alpha \in \mathcal{P}_{1}(n)$ (i.e. even rows have even multiplicity);

(2) $\epsilon: \mathbb{Z}_{\geqslant 0} \rightarrow\{\omega, 0,1\}$.

The function $\epsilon$ is required to satisfy, for all $i \geqslant 0$ :

$$
\epsilon(i)= \begin{cases}\omega, & \text { if } i \text { even; } \\ \omega, & \text { if } m_{\alpha}(i)=0 \\ 1, & \text { if } i \text { odd, } m_{\alpha}(i) \text { odd; } \\ 0 \text { or } 1, & \text { if } i \text { odd }, m_{\alpha}(i)>0 \text { even. }\end{cases}
$$

Proposition 2.4. If $\underline{p}=2$, the unipotent conjugacy classes of $\mathrm{GL}^{\dagger}(n)$ in $\mathrm{GL}^{\dagger}(n) \backslash \mathrm{GL}(n)$ are parametrized by $\widetilde{\mathcal{P}_{1}}(n)$.

We write $\mathcal{U}_{\alpha, \epsilon}$ for the unipotent class parametrized by $(\alpha, \epsilon)$. 
The map from unipotent classes in $G_{1}$ to parameters is defined as follows. Define the map $S: G_{1} \rightarrow G_{0}$ to be $S(\phi)=\phi^{2}$. If $\phi$ is unipotent then so is $S(\phi)$, and therefore $S(\phi)$ defines a partition $\alpha$ of $n$, and it is easy to see $\alpha \in \mathcal{P}_{1}(n)$. Suppose $i$ is odd and $m_{\alpha}(i)>0$ is even. Then define $\epsilon(i)=0$ if $f\left(v,(g-1)^{i-1} v\right)=0$ for all $v \in \operatorname{ker}(g-1)^{i}$, and $\epsilon(i)=1$ otherwise.

2.3. Closure Relations. We now describe the closure relations on unipotent classes. As discussed in Section 2.1 if the characteristic is good then the closure relations on classes are given by the order relation on partitions. So assume $p=2$ and $G=\mathrm{GL}^{\dagger}, S p(2 n)$ or $O(2 n)$.

We define a partial order on the sets $\widetilde{\mathcal{P}_{-1}}(2 n)$ and $\widetilde{\mathcal{P}_{1}}(n)$ defined in the previous section. Suppose $(\alpha, \epsilon)$ and $(\beta, \delta)$ are elements of one of these sets. We say $(\alpha, \epsilon) \leqslant(\beta, \delta)$ if

(1) $\alpha \leqslant \beta \quad\left(\Leftrightarrow \alpha^{*} \geqslant \beta^{*}\right)$;

and for all $k \geqslant 1$ :

(2) $\left(\sum_{i=1}^{k} \beta_{i}^{*}\right)-\max \left(\delta_{k}, 0\right) \leqslant\left(\sum_{i=1}^{k} \alpha_{i}^{*}\right)-\max \left(\epsilon_{k}, 0\right)$;

(3) If $\sum_{i=1}^{k} \alpha_{i}^{*}=\sum_{i=1}^{k} \beta_{i}^{*}$ and $\alpha_{k+1}^{*}-\beta_{k+1}^{*}$ is odd then $\delta_{k} \neq 0$.

Proposition 2.5. Suppose $p=2$ and $G=\mathrm{GL}^{\dagger}(n), S p(2 n)$ or $O(2 n)$. Suppose $(\alpha, \epsilon),(\beta, \delta)$ are both in $\widetilde{\mathcal{P}_{1}}(n), \widetilde{\mathcal{P}_{-1}}(2 n)$, or $\widetilde{\mathcal{P}_{-1}}(2 n)$, respectively. In the case of $O(2 n)$ assume they are both in $\widetilde{\mathcal{P}_{-1}}(2 n)_{0}$ or $\widetilde{\mathcal{P}_{-1}}(2 n)_{1}$.

Then $\mathcal{U}_{\alpha, \epsilon} \preceq_{u} \mathcal{U}_{\beta, \delta}$ if and only if $(\alpha, \epsilon) \leqslant(\beta, \delta)$.

Corollary 2.6. Fix a partition $\alpha$ of the appropriate type for $G$. Then the set $\left\{\mathcal{U}_{\alpha, \epsilon}\right\}$ (as $\epsilon$ varies) has a unique maximal element.

Proof. Recall $\epsilon(i) \in\{\omega, 0,1\}$, and is determined by $\alpha$ except in some cases when it can be 0 or 1 ; for $\epsilon_{\max }$ always choose 1 whenever there is a choice.

Explicitly, define:

$$
\epsilon_{\max }^{\alpha}(i)= \begin{cases}1, & \text { if } \quad i>0 \text { odd, } m_{\alpha}(i)>0 \text { even and } \alpha \in \widetilde{\mathcal{P}_{1}}(n) ; \\ 1, & \text { if } \quad i>0 \text { even, } m_{\alpha}(i)>0 \text { even and } \alpha \in \widetilde{\mathcal{P}_{-1}}(2 n) .\end{cases}
$$

This determines $\epsilon_{\max }^{\alpha}$ completely, and the result is immediate from Proposition 2.5 .

\section{LUSZTIG'S MAP FOR CLASSICAL GROUPS}

3.1. The function $\psi$. We recall a function defined in [Lu11, §1.6].

Suppose $\alpha=\left(\alpha_{1}, \ldots, \alpha_{\ell}\right)$ is a partition. Define a function

$$
\psi_{\alpha}:\{i \mid 1 \leqslant i \leqslant \ell\} \rightarrow\{-1,0,1\}
$$

as follows. Set $\alpha_{0}=\alpha_{\ell+1}=0$.

(1) If $i$ is odd and $\alpha_{i-1}>\alpha_{i}$ then $\psi(i)=1$;

(2) If $i$ is even and $\alpha_{i}>\alpha_{i+1}$ then $\psi(i)=-1$;

(3) In all other cases $\psi(i)=0$.

This satisfies some obvious properties.

For $\ell \in \mathbb{Z}$ define $\kappa_{\ell} \in\{0,1\}$ by $(-1)^{\ell}=(-1)^{\kappa_{\ell}}$. Then 
(4) $\psi_{\alpha}(1)=1 ; \psi_{\alpha}(\ell)=-1$ if $\ell$ is even;

(5) If $k \leqslant \ell$ is odd then $\sum_{i=1}^{k} \psi_{\alpha}(i)=1$;

(6) If $k \leqslant \ell$ is even then $\sum_{i=1}^{k} \psi_{\alpha}(i)=1+\psi_{\alpha}(k)$;

(7) $\sum_{i=1}^{\ell} \psi_{\alpha}(i)=\kappa_{\ell}$.

Suppose $\alpha$ is a partition of $n$, and all entries $\alpha_{i}$ of $\alpha$ are even. Then let

$$
\alpha+\psi_{\alpha}=\left(\alpha_{1}+\psi_{\alpha}(1), \ldots, \alpha_{\ell}+\psi_{\alpha}(\ell)\right) .
$$

This is a partition of $n+\kappa_{\ell}$. Explicitly, if we write $\alpha+\psi_{\alpha}=\left(\alpha_{1}^{\prime}, \ldots, \alpha_{\ell}^{\prime}\right)$, and if $2 i+1 \leqslant \ell$, then

$$
\left(\alpha_{2 i}^{\prime}, \alpha_{2 i+1}^{\prime}\right)= \begin{cases}\left(\alpha_{2 i}, \alpha_{2 i+1}\right), & \text { if } \alpha_{2 i}=\alpha_{2 i+1} \\ \left(\alpha_{2 i}-1, \alpha_{2 i+1}+1\right), & \text { if } \alpha_{2 i}>\alpha_{2 i+1} .\end{cases}
$$

Also $\alpha_{1}^{\prime}=\alpha_{1}+1$ and, if $\ell$ is even, $\alpha_{\ell}^{\prime}=\alpha_{\ell}-1$.

3.2. Parametrization of elliptic conjugacy classes. For classical groups the elliptic conjugacy classes in $W$ are parametrized as follows. Recall $\mathcal{P}(n)$ is the set of partitions of $n, \mathcal{P}(n)_{0}$ is the subset of $\mathcal{P}(n)$ consisting of partitions with an even number of parts, and $\mathcal{P}(n)^{\text {odd }}$ is the set of partitions of $n$ consisting of only odd parts.

(1) $G=G^{\dagger}(n)$. Then $G$ has two connected components: $G^{0}=G L(n)$ and $D=G \backslash G^{0}$. We have

- $\left[W_{e}^{G^{0}}\right]$ is a singleton, and the only element is the conjugacy class of Coxeter elements;

- $\left[W_{e}^{D}\right]$ is parametrized by $\mathcal{P}^{o d d}(n)$.

(2) $G=S O(2 n+1)$ or $S p(2 n)$ : [ $\left.W_{e}\right]$ is parametrized by $\mathcal{P}(n)$.

(3) $G=O(2 n)$. Then $G$ has two connected components: $G^{0}=S O(2 n)$ and $D=G \backslash G^{0}$.

- $\left[W_{e}\right]$ is parametrized by $\mathcal{P}(n)$;

- $\left[W_{e}^{G^{0}}\right]$ is parametrized by $\mathcal{P}(n)_{0}$;

- $\left[W_{e}^{D}\right]$ is parametrized by $\mathcal{P}(n)_{1}$.

For $\alpha \in \mathcal{P}(n)$, we write $\mathcal{C}_{\alpha}$ for the corresponding elliptic conjugacy class in $W$.

3.3. Explicit description of $\Phi$ for classical groups. Suppose $G$ is a classical group. Lusztig's map $\Phi_{e}:\left[W_{e}^{D}\right] \rightarrow\left[D_{u}\right]$ is described explicitly in $[\mathrm{Lu} 11, \S 4.2]$ and [Lu12b, §3.7 \& §5.5].

(1) $G=\mathrm{GL}^{\dagger}(n)$. In this case, $\Phi_{e}$ sends the conjugacy class of Coxeter elements to the principal unipotent class. Note that both the conjugacy class of Coxeter elements and the principal unipotent class correspond to the partition $(n)$ of $n$.

If $p=2$, then $D$ contains unipotent elements. In this case, the map $\Phi_{e}$ : $\left[W_{e}^{D}\right] \rightarrow\left[D_{u}\right]$ is given by $\mathcal{C}_{\alpha} \mapsto \mathcal{U}_{\alpha, \epsilon_{\max }^{\alpha}}$ for $\alpha \in \mathcal{P}^{\text {odd }}(n)$.

(2) $G=O(2 n+1)$. The elliptic conjugacy classes of $W$ are parametrized by $\mathcal{P}(n)$. 
(i) $p \neq 2$ : the map $\Phi_{e}$ is given by $\mathcal{C}_{\alpha} \mapsto \mathcal{U}_{\alpha^{\prime}}$, where

$$
\alpha^{\prime}= \begin{cases}2 \alpha+\psi_{\alpha}, & \text { if } \alpha \text { has an odd number of parts } \\ \left(2 \alpha+\psi_{\alpha}, 1\right), & \text { if } \alpha \text { has an even number of parts. }\end{cases}
$$

(ii) $p=2$ : the map $\Phi_{e}$ is given by $\mathcal{C}_{\alpha} \mapsto \mathcal{U}_{\alpha^{\prime}, \epsilon_{\text {max }}^{\alpha^{\prime}}}$ where $\alpha^{\prime}=(2 \alpha, 1)$.

(3) $G=S p(2 n)$. The elliptic conjugacy classes of $W$ are parametrized by $\mathcal{P}(n)$.

(i) $p \neq 2$ : the map $\Phi_{e}$ is given by $\mathcal{C}_{\alpha} \mapsto \mathcal{U}_{2 \alpha}$.

(ii) $p=2$ : the map $\Phi_{e}$ is given by $\mathcal{C}_{\alpha} \mapsto \mathcal{U}_{\left(2 \alpha, \epsilon_{\text {max }}^{2 \alpha}\right)}$.

(4) $G=O(2 n)$.

If $p \neq 2$, then the unipotent elements of $G$ are contained in $G^{0}=S O(2 n)$. In this case, $\left[W^{G^{0}}\right]$ is parametrized by $\mathcal{P}(n)_{0}$. The map $\Phi_{e}:\left[W_{e}^{G^{0}}\right] \rightarrow\left[G_{u}^{0}\right]$ is given by $\mathcal{C}_{\alpha} \mapsto \mathcal{U}_{2 \alpha+\psi_{\alpha}}$.

If $p=2$, then $\left[W_{e}\right]$ is parametrized by $\mathcal{P}(n)$ and the map $\Phi_{e}:\left[W_{e}\right] \rightarrow$ $\left[G_{u}\right]$ is given by $\mathcal{C}_{\alpha} \mapsto \mathcal{U}_{2 \alpha, \epsilon_{\max }^{2 \alpha}}$. On the other hand $\left[W_{e}^{D}\right]$ is parametrized by $\mathcal{P}(n) \backslash \mathcal{P}(n)_{0}$, and the map $\Phi_{e}:\left[W_{e}^{D}\right] \rightarrow\left[D_{u}\right]$ is $\mathcal{C}_{\alpha} \mapsto \mathcal{U}_{2 \alpha, \epsilon_{\max }^{2 \alpha}}$.

Note that each unipotent class $\mathcal{U}_{2 \alpha, \epsilon_{\max }^{2 \alpha}}$ is a single $G^{0}$-conjugacy classes.

3.4. Map from characteristic 0 to characteristic 2. For the moment let $G_{p}$ be a connected reductive group, defined over an algebraically closed field $\mathbb{F}$ of characteristic $p>0$. Let $G_{0}$ be the complex group with the same root datum as $G$. Consider the sets $\left[G_{p, u}\right]$ and $\left.\left[G_{0, u}\right]\right)$ of unipotent conjugacy classes of $G_{p}$ and $G_{0}$, respectively.

Proposition 3.1. [Sp82, Theorem III.5.2] There is an injective, dimension preserving map $\pi_{p}:\left[G_{0, u}\right] \rightarrow\left[G_{p, u}\right]$, such that $\pi_{p}$ is an isomorphism of partially ordered sets from $\left[G_{0, u}\right]$ to its image.

Lusztig gives an alternative description of this map in terms of the Springer correspondence [Lu11, §4.1], and shows in [Lu12a, Theorem 0.4 and §3.9] that the following diagram is commutative:

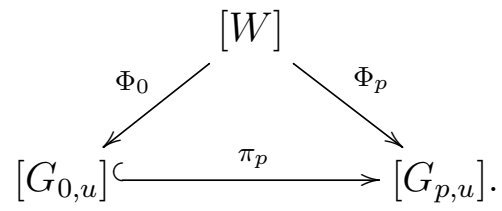

We now assume $G$ is a classical group. In this case $\pi_{p}$ is a bijection for $p \neq 2$. In the table below, we list the sets parametrizing the objects in types $B, C, D$, over $\mathbb{C}$ and in characteristic 2.

\begin{tabular}{lll}
$G$ & $\mathbb{C}$ & $\overline{\mathbb{F}_{2}}$ \\
\hline$S p(2 n)$ & $\mathcal{P}_{-1}(2 n)$ & $\widetilde{\mathcal{P}_{-1}}(2 n)$ \\
$O(2 n+1)$ & $\mathcal{P}_{1}(2 n+1)$ & $\widetilde{\mathcal{P}_{-1}}(2 n)$ \\
$O(2 n)$ & $\mathcal{P}_{1}(2 n)$ & $\widetilde{\mathcal{P}_{-1}}(2 n)$
\end{tabular}


We have the following diagram

$$
\left[G_{0, u}\right] \underset{\theta_{2}}{\stackrel{\pi_{2}}{\rightleftarrows}}\left[G_{2, u}\right],
$$

where $\theta_{2} \circ \pi_{2}=$ id. Both $\pi_{2}, \theta_{2}$ are defined explicitly in [Sp82, III, $\S 6-8$ ].

Implicit in the statement that Spaltenstein's and Lusztig's definitions of $\pi_{2}$ agree is the following result. This makes the relationship between Lusztig's function $\psi_{\alpha}$ and Spaltenstein's map $\theta_{2}$ precise in the case of classical groups.

Lemma 3.2. Suppose $G$ is of type $B, C$ or $D$, and $\mathcal{U} \in \Phi_{2}\left[W_{e}\right]$. Write $\mathcal{U}=$ $\mathcal{U}_{\left(\alpha, \epsilon_{\text {max }}^{\alpha}\right)}$ as in Section 3.3. Then

$$
\theta_{2}\left(\mathcal{U}_{\left(\alpha, \epsilon_{\text {max }}^{\alpha}\right)}\right)= \begin{cases}\mathcal{U}_{\alpha} & \text { type } C \\ \mathcal{U}_{\alpha+\psi_{\alpha}} & \text { types } B, D\end{cases}
$$

Thus the map $\theta_{2}$, which is inverse to $\pi_{2}$, has a simple description when restricted to the image of the elliptic elements.

Proof. This is immediate in the case of $\operatorname{Sp}(2 n)$ : by [Sp82, III, 6.1] $\theta_{2}\left(\mathcal{U}_{\alpha, \epsilon_{\max }^{\alpha}}\right)=\mathcal{U}_{\alpha}$.

Suppose $G=\mathrm{SO}(2 n)$ and $\beta \in \mathcal{P}(n)$. Then $\Phi\left(\mathcal{C}_{\beta}\right)=\mathcal{U}_{\left(2 \beta, \epsilon_{\max }^{2 \beta}\right)}$. So suppose $\alpha \in \mathcal{P}(2 n)$ has all even parts.

Define $\delta$ by [Sp82, III, Lemma 7.3], so that

$$
\theta_{2}\left(\mathcal{U}_{\left(\alpha, \epsilon_{\max }^{\alpha}\right)}\right)=\mathcal{U}_{\delta(\alpha)}
$$

Set $\gamma=\alpha+\psi_{\alpha}$, so we have to show $\delta=\gamma$.

According to [Sp82, Lemma 7.3], and using the definition of $\epsilon_{\max }^{\alpha}$, we see $\epsilon_{\max }^{\alpha}(i)=$ 1 if and only if there is a row of length $i$, i.e. $m_{\alpha}(i)>0$. Given this we see

$$
\begin{aligned}
\delta_{i}^{*}=\alpha_{i}^{*}+1, & \text { if } i \text { is odd, } \alpha_{i}^{*} \text { is even, } m_{\alpha}(i-1)>0 ; \\
\delta_{i}^{*}=\alpha_{i}^{*}-1, & \text { if } i \text { is even, } \alpha_{i}^{*} \text { is even, } m_{\alpha}(i)>0 ; \\
\delta_{i}^{*}=\alpha_{i}, & \text { otherwise. }
\end{aligned}
$$

On the other hand

$$
\begin{array}{cc}
\gamma_{j}=\alpha_{j}+1, & \text { if } j \text { is odd, } \alpha_{j-1}>\alpha_{j} \\
\gamma_{j}=\alpha_{j}-1, & \text { if } j \text { is even, } \alpha_{j}>\alpha_{j+1} \\
\gamma_{j}=\alpha_{j}, & \text { otherwise. }
\end{array}
$$

There is a bijection:

$$
\left\{i \text { odd } \mid \alpha_{i}^{*} \text { is even, } m_{\alpha}(i-1)>0\right\} \leftrightarrow\left\{j \text { odd } \mid \alpha_{j-1}>\alpha_{j}\right\} .
$$

The bijection takes $i \mapsto j=\alpha_{i}^{*}+1$, with inverse $j \mapsto i=\alpha_{j}+1$. Under this bijection incrementing $\alpha_{i}^{*}$ by 1 corresponding to incrementing $\alpha_{j}$ by 1 . Similarly there is a bijection

$$
\left\{i \text { even } \mid \alpha_{i}^{*} \text { is even, } m_{\alpha}(i)>0\right\} \leftrightarrow\left\{j \text { even } \mid \alpha_{j}>\alpha_{j+1}\right\},
$$

taking $i \mapsto j=\alpha_{i}^{*}$ and $j \mapsto i=\alpha_{j}$, under which decreasing $\alpha_{i}$ by 1 corresponds to decreasing $\alpha_{j}^{*}$ by 1 . This completes the proof for $\mathrm{SO}(2 n)$. We leave the very similar case of $\mathrm{SO}(2 n+1)$ to the reader. 
Example 3.3. Suppose $G=\mathrm{SO}(2 n)$ and $\alpha=[6,6,4,2]$. Then $\alpha^{*}=[4,4,3,3,2,2]$. Thus $\delta_{1}^{*}=\alpha_{1}^{*}=4, \delta_{2}^{*}=\alpha_{2}^{*}-1=3, \delta_{3}^{*}=\alpha_{3}^{*}=3, \delta_{4}^{*}=\alpha_{4}^{*}=3, \delta_{5}^{*}=\alpha_{5}^{*}+1=3, \delta_{6}^{*}=$ $\alpha_{6}^{*}-1=1, \delta_{7}^{*}=\alpha_{7}^{*}+1=1$. This gives the partition $\delta^{*}=[4,3,3,3,3,1,1]$, or $\delta=[7,5,5,1]$.

On the other hand $\gamma_{1}=\alpha_{1}+1=7, \gamma_{2}=\alpha_{2}-1=5, \gamma_{3}=\alpha_{3}+1=5, \gamma_{4}=$ $\alpha_{4}-1=1$, so $\gamma=[7,5,5,1]=\delta$.

Returning to Proposition 3.1, this gives a reduction of Theorem 1.1 in the classical case to characteristic 2 , in which case the explicit description of $\Phi_{2}$ restricted to $\left[W_{e}\right]$ is quite simple. We have the following result.

Proposition 3.4. Let $G$ be a connected classical group defined over an algebraically closed field of characteristic $p \geqslant 0$. Let $\mathcal{C}_{\alpha}, \mathcal{C}_{\beta} \in\left[W_{e}\right]$, parametrized by certain partitions as in Section 3.2. Then $\Phi\left(\mathcal{C}_{\alpha}\right) \preceq{ }_{u} \Phi\left(\mathcal{C}_{\beta}\right)$ if and only if $\alpha \leqslant \beta$.

The same statement holds for $\mathrm{GL}^{\dagger}(n)$ and $\mathrm{O}(n)$, with $\mathcal{C}_{\alpha}, \mathcal{C}_{\beta} \in\left[W_{e}^{D}\right]$ and $\Phi_{e}$ : $\left[W_{e}^{D}\right] \mapsto\left[D_{u}\right]$.

Proof. If $p \neq 2$, then $\left[G_{p, u}\right]$ and $\left[G_{0, u}\right]$ are the same as partially ordered sets. Moreover, the map $\pi_{2}:\left[G_{0, u}\right] \rightarrow\left[G_{2, u}\right]$ is an isomorphism from the poset $\left[G_{0, u}\right]$ to its image. In other words, $\Phi_{p}\left(\mathcal{C}_{\alpha}\right) \preceq_{u} \Phi_{p}\left(\mathcal{C}_{\beta}\right)$ if and only if $\Phi_{2}\left(\mathcal{C}_{\alpha}\right) \preceq_{u} \Phi_{2}\left(\mathcal{C}_{\beta}\right)$, where $\Phi_{p}$ is Lusztig's map for $G$ in characteristic $p$.

Now by $\S 3.3$, for $G=S p(2 n)$ or $O(n)$, the map $\Phi_{2}$ is given by $\mathcal{C}_{\alpha} \mapsto \mathcal{U}_{\alpha^{\prime}, \epsilon_{\max }^{\alpha^{\prime}}}$ where $\alpha^{\prime}=2 \alpha$ or $(2 \alpha, 1)$. By $\S 2.3, \mathcal{U}_{\alpha^{\prime}, \epsilon_{\max }^{\alpha^{\prime}}} \leqslant \mathcal{U}_{\beta^{\prime}, \epsilon_{\max }^{\beta^{\prime}}}$ if and only if $\alpha^{\prime} \leqslant \beta^{\prime}$, which is equivalent to $\alpha \leqslant \beta$.

In the twisted cases $\mathrm{GL}^{\dagger}(n)$ and $\mathrm{O}(2 n)$ we only have to consider the case $p=2$, in which case $\Phi_{2}$ is given by $\alpha \mapsto\left(\alpha, \epsilon_{\max }^{\alpha}\right)$ or $\left(2 \alpha, \epsilon_{\max }^{2 \alpha}\right)$ respectively, and the same argument applies.

Using this Theorem 0.1 for classical groups is equivalent to the following combinatorial statement purely about the Weyl group.

Proposition 3.5. Suppose $W$ is a classical Weyl group. Suppose $\mathcal{C}_{\alpha}, \mathcal{C}_{\beta} \in\left[W_{e}^{D}\right]$, for partitions $\alpha, \beta$ as in Section 3.2. Then

$$
\mathcal{C}_{\alpha} \preceq_{W} \mathcal{C}_{\beta} \Leftrightarrow \beta \leqslant \alpha .
$$

Proof of Theorem 0.1 for classical groups. Then by Propositions 3.4 and 3.5,

$$
\Phi\left(\mathcal{C}_{\alpha}\right) \preceq_{u} \Phi\left(\mathcal{C}_{\beta}\right) \Leftrightarrow \alpha \leqslant \beta \Leftrightarrow \mathcal{C}_{\beta} \preceq_{W} \mathcal{C}_{\alpha},
$$

which implies Theorem 1.1 for classical groups.

We prove Proposition 3.5 in Section 5.

\section{EXAMPLES}

We explicitly describe the maps from $[W]$ to $\left[G(\mathbb{C})_{u}\right]$ and $\left[G\left(\overline{\mathbb{F}}_{2}\right)_{u}\right]$ for some classical groups of small rank.

The elliptic conjugacy classes in $W$ are parametrized by partitions as discussed above. General conjugacy classes in $W$ are parametrized by a pair $\left(L, \mathcal{C}_{L}\right)$ where $L$ is a Levi factor and $\mathcal{C}_{L}$ is an elliptic conjugacy class in $L$ [GP00]. When $L$ is of type $A$ it only has one elliptic conjugacy class so we drop it from the notation.

In each table the elliptic classes are listed first, followed by a line, and then the non-elliptic classes. 
The unipotent orbits in $G(\mathbb{C})$ are parametrized by certain partitions, and the unipotent orbits in $G\left(\overline{\mathbb{F}_{2}}\right)$ are parametrized by pairs, as described in Section 2.

4.1. $\operatorname{Sp}(4)$. Here $[W] \stackrel{\Phi_{2}}{\longrightarrow}\left[S p\left(4, \overline{\mathbb{F}_{2}}\right)_{u}\right]$ is a bijection (both sets have 5 elements), whereas $\left[\operatorname{Sp}(4, \mathbb{C})_{u}\right]$ has 4 elements.

\begin{tabular}{lll}
{$[W]$} & $S p(4, \mathbb{C})$ & $\operatorname{Sp}\left(4, \overline{\mathbb{F}}_{2}\right)$ \\
\hline \hline$[2]$ & {$[4]$} & $([4], *)$ \\
{$[1,1]$} & {$[2,2]$} & $([2,2], \epsilon(2)=1)$ \\
\hline$A_{1}^{s}$ & {$[2,2]$} & $([2,2], \epsilon(2)=0)$ \\
$A_{1}^{l}$ & {$[2,1,1]$} & $([2,1,1], *)$ \\
$T$ & {$[1,1,1,1]$} & $([1,1,1,1], *)$
\end{tabular}

4.2. $\mathrm{SO}(4)$.

The unipotent orbits in $\mathrm{SO}\left(4, \overline{\mathbb{F}}_{2}\right)$ are parametrized by $\widetilde{\mathcal{P}_{-1}}(4)_{0}$ (odd rows have even multiplicity, the first column has even length). Also a strongly even partitions count twice provided $\epsilon(i)=0$ for all $i$; these are denote with a subscript $I$ or $I I$.

There is one elliptic class in $W$ and 4 overall. In this case all three sets are in bijection.

\begin{tabular}{lll}
{$[W]$} & $S O(4, \mathbb{C})$ & $\mathrm{SO}\left(4, \overline{\mathbb{F}}_{2}\right)$ \\
\hline \hline$[1,1]$ & {$[3,1]$} & $([2,2], \epsilon(2)=1)$ \\
\hline$\left(A_{1}, *\right)$ & {$[2,2]_{I}$} & $([2,2], \epsilon(2)=0)_{I}$ \\
$\left(A_{1}^{\prime}, *\right)$ & {$[2,2]_{I I}$} & $([2,2], \epsilon(2)=0)_{I I}$ \\
$(T, *)$ & {$[1,1,1,1]$} & $([1,1,1,1], *)$
\end{tabular}

4.3. $\mathrm{SO}(6)$.

The Weyl group of $\mathrm{SO}(6)$ is of type $D_{3}$ which has 5 conjugacy classes, 1 of which is elliptic.

\begin{tabular}{lll}
{$[W]$} & $S O(6, \mathbb{C})$ & $\mathrm{SO}\left(6, \overline{\mathbb{F}}_{2}\right)$ \\
\hline \hline$[2,1]$ & {$[5,1]$} & $([4,2], *)$ \\
\hline$\left(A_{2}, *\right)$ & {$[3,3]$} & $([3,3], *)$ \\
$\left(D_{2}, *\right)$ & {$[3,1,1,1]$} & $([2,2,1,1], \epsilon(2)=1)$ \\
$\left(A_{1}, *\right)$ & {$[2,2,1,1]$} & $([2,2,1,1], \epsilon(2)=0)$ \\
$(T, *)$ & {$[1,1,1,1,1,1]$} & $([1,1,1,1,1,1], *)$
\end{tabular}

4.4. $\mathrm{SO}(8)$.

The Weyl group of $\mathrm{SO}(8)$ is of type $D_{3}$, which has 13 conjugacy classes, 3 of which are elliptic. Both $\mathrm{SO}(8, \mathbb{C})$ and $\mathrm{SO}\left(8, \overline{\mathbb{F}_{2}}\right)$ have 12 unipotent classes. Note that the orbits $[3,2,2,1]$ and $([2,2,2,2], \epsilon(2)=1)$ both occur twice in the image.

Note that $S O(8)$ has two non-conjugate $G L(4)=A_{3}$ Levi factors in addition to $G L(1) \times S O(6)$ of type $D_{3}$. Also it has two non-conjugate $\mathrm{GL}(2) \times \mathrm{GL}(2)$ factors. 
Also a unipotent orbit for $\mathrm{O}\left(8, \overline{\mathbb{F}_{2}}\right)$ splits into two for $\mathrm{SO}\left(8, \overline{\mathbb{F}_{2}}\right)$ if and only if all parts are even, with even multiplicity, and all $\epsilon(i)=0$.

\begin{tabular}{lll}
{$[W]$} & $S O(8, \mathbb{C})$ & $\mathrm{SO}\left(8, \overline{\mathbb{F}}_{2}\right)$ \\
{$[3,1]$} & {$[7,1]$} & $([6,2], *)$ \\
{$[2,2]$} & {$[5,3]$} & $([4,4], \epsilon(4)=1)$ \\
{$[1,1,1,1]$} & {$[3,2,2,1]$} & $([2,2,2,2], \epsilon(2)=1)$ \\
\hline$D_{3}=S O(6) \times G L(1)$ & {$[5,1,1,1]$} & {$[4,2,1,1]$} \\
$A_{3}=G L(4)$ & {$[4,4]_{I}$} & $([4,4], \epsilon(4)=0)_{I}$ \\
$A_{3}^{\prime}=G L(4)^{\prime}$ & {$[4,4]_{I I}$} & $([4,4], \epsilon(4)=0)_{I I}$ \\
$A_{1} \times D_{2}=G L(2) \times S O(4)$ & {$[3,2,2,1]$} & $([2,2,2,2], \epsilon(2)=1)$ \\
$D_{2}=\mathrm{GL}(1) \times \mathrm{SO}(4)$ & {$[3,1,1,1,1,1]$} & $([2,2,1,1,1,1], \epsilon(2)=1)$ \\
$A_{2}=\mathrm{GL}(3) \times \mathrm{GL}(1)$ & {$[3,3,1,1]$} & $([3,3,1,1], *)$ \\
$2 A_{1}=\mathrm{GL}(2) \times \mathrm{GL}(2)$ & {$[2,2,2,2]_{I}$} & $([2,2,2,2], \epsilon(2)=0)_{I}$ \\
$2 A_{1}=\mathrm{GL}(2) \times \mathrm{GL}(2)^{\prime}$ & {$[2,2,2,2]_{I I}$} & $([2,2,2,2], \epsilon(2)=0)_{I I}$ \\
$A_{1}=\mathrm{GL}(2) \times \mathrm{GL}(1) \times \mathrm{GL}(1)$ & {$[2,2,1,1,1,1]$} & $([2,2,1,1,1,1,1,1], \epsilon(2)=0)$ \\
$T$ & {$\left[1^{8}\right]$} & $\left(\left[1^{8}\right], *\right)$
\end{tabular}

4.5. $\mathrm{SO}(12)$.

We only discuss the elliptic classes.

$\begin{array}{lll}{[W]} & S O(12, \mathbb{C}) & \mathrm{SO}\left(12, \overline{\mathbb{F}}_{2}\right) \\ {[5,1]} & {[11,1]} & ([10,2], *) \\ {[4,2]} & {[9,3]} & ([8,4], *) \\ {[3,3]} & {[7,5]} & ([6,6], \epsilon(6)=1)) \\ {[2,2,1,1]} & {[5,3,3,1]} & ([4,4,2,2], \epsilon(4)=\epsilon(2)=1) \\ {[1,1,1,1,1,1]} & {[3,2,2,2,2,1]} & ([2,2,2,2,2,2], \epsilon(2)=1)\end{array}$

\section{Proof of the Main Theorem for Classical groups}

We start by giving a version of the Bruhat order for classical groups which is convenient for our purposes.

If $G=G L(n)$, then $W \simeq S_{n}$. For $w \in W$ and $1 \leqslant i, j \leqslant n$, we define

$$
w\{i, j\}=\{1 \leqslant k \leqslant i \mid w(k) \geqslant j\}, \quad w[i, j]=|w\{i, j\}| .
$$

If $G=S O(2 n+1)$ or $S p(2 n)$ or $O(2 n)$, then $W \cong S_{n} \ltimes(\mathbb{Z} / 2 \mathbb{Z})^{n}$. We identify $W$ with the set of permutations $\sigma$ of $\{ \pm 1, \pm 2, \ldots, \pm n\}$ satisfying $\sigma(-i)=-\sigma(i)$ for all $i$. For $-n \leqslant i, j \leqslant n$, we define

$$
w\{i, j\}=\{-n \leqslant k \leqslant i, w(k) \geqslant j\}, \quad w[i, j]=|w\{i, j\}| .
$$

We use the following labeling on the Dynkin diagrams. 


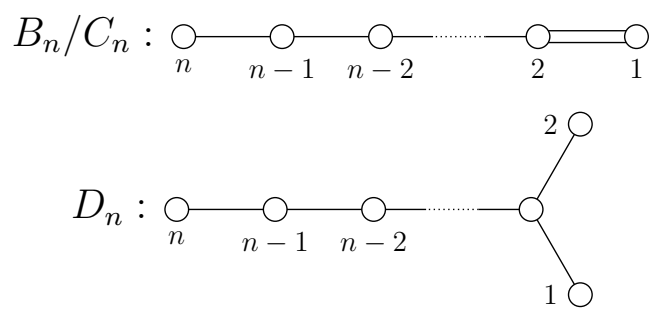

Proposition 5.1. Suppose $W$ is simple and classical, and $x, y \in W$.

(1) If $G=G L(n)$, then $x \leqslant y \Leftrightarrow x[i, j] \leqslant y[i, j]$ for all $1 \leqslant i, j \leqslant n$;

(2) If $G=S O(2 n+1)$ or $S p(2 n)$, then $x \leqslant y \Leftrightarrow x[i, j] \leqslant y[i, j]$ for all $-n \leqslant$ $i, j \leqslant n$;

(3) If $G=O(2 n)$, then $x \leqslant y \Rightarrow x[i, j] \leqslant y[i, j]$ for all $-n \leqslant i, j \leqslant n$.

Remark 5.2. It is worth pointing out that although the Weyl group of $O(2 n)$ is isomorphic to the Weyl group of $S O(2 n+1)$ and $S p(2 n)$, the simple reflections and thus the Bruhat order are different. In particular, the explicit description of the Bruhat order for the Weyl group of $O(2 n)$ is more complicated than that of $S O(2 n+1)$ or $S p(2 n)$ and we only use a weak version of the Bruhat order for the Weyl group of $O(2 n)$ here. The readers interested in the full explicit description may refer to [BB05].

Part (1) is proved in [BB05, Theorem 2.1.5]. Part (2) is proved in [BB05, Theorem 8.1.8]. One may also argue as follows. In [Lu03], Lusztig showed that the subgroup of a Coxeter group which is the fixed points of the action of a diagram automorphism is again a Coxeter group and the Bruhat order of these two groups are compatible. Thus part (2) may be deduced directly part (1). As to part (3), if $x, y \in W(S O(2 n))$, then the statement follows from [BB05, Theorem 8.2.8]. The general case follows from part (2) and the following Lemma.

Lemma 5.3. We identify the (extended) Weyl group of $O(2 n)$ with the Weyl group of $S p(2 n)$ as the abstract group $W=S_{n} \ltimes(\mathbb{Z} / 2 \mathbb{Z})^{n}$ in the natural way. We denote by $\leqslant^{D}$ and $\leqslant^{C}$ the Bruhat order for $W(O(2 n))$ and $W(S p(2 n))$ respectively. Let $x, y \in W$. If $x \leqslant^{D} y$, then $x \leqslant^{B} y$.

Proof. It suffices to consider the case where $y=x s_{\alpha}$ for some positive root $\alpha$ of $S O(2 n)$. In this case, $x \leqslant^{D} y$ is equivalent to say that $x(\alpha)$ is a positive root of $S O(2 n)$. Under the identification of $W(O(2 n))$ with $W(S p(2 n))$ as abstract groups, we may identify the set of positive roots of $S O(2 n)$ as a subset of the set of positive roots of $S p(2 n)$ ). In particular, $x(\alpha)$ is also a positive root of $S p(2 n)$. Thus $x \leqslant^{B} y$.

The remainder of this section is concerned with the proof of Proposition 3.5.

Following $[\mathrm{He} 07]^{1}$, for $1 \leqslant a, b \leqslant n$, we define

$$
s_{[a, b]}= \begin{cases}s_{a} s_{a+1} \cdots s_{b}, & \text { if } a \leqslant b \\ 1, & \text { otherwise }\end{cases}
$$

\footnotetext{
${ }^{1}$ Note that the convention for the labeling on the simple reflections here is opposite to the labeling used in $[\mathrm{He} 07]$ and the formulas below are modified accordingly.
} 
5.1. Case 1: Type $B_{n} / C_{n} / D_{n}$. Let $\alpha=\left(\alpha_{1}, \alpha_{2}, \ldots, \alpha_{\ell}\right)$ be a partition of $n$. The corresponding class $\mathcal{C}_{\alpha} \in\left[W_{e}\right]$ consists of the permutations $w$ satisfying:

(1) There exists a decomposition $\{1,2, \ldots, n\}=I_{1} \sqcup I_{2} \sqcup \ldots \sqcup I_{l}$;

(2) $\left|I_{j}\right|=a_{j}$ for all $j$;

(3) The orbits of $w$ on $\{ \pm 1, \ldots, \pm n\}$ are $I_{j} \sqcup-I_{j}$ for $1 \leqslant j \leqslant l$.

We have the following useful inequalities for the elements in $\mathcal{C}_{\alpha}$.

Proposition 5.4. Suppose $w \in \mathcal{C}_{\alpha}$ and $0 \leqslant m \leqslant n$. Then

$$
w[n-m, n-m+1] \geqslant \min \left\{k ; a_{1}+\cdots+a_{k} \geqslant m\right\} .
$$

Proof. Let $I_{1}, \ldots, I_{l}$ be the subsets of $\{1, \ldots, n\}$ satisfying the conditions (1)-(3) for $w$. Let $1 \leqslant j \leqslant l$ and $r=\max \left\{i \mid i \in I_{j}\right\}$. Then $w^{\left|I_{j}\right|}(-r)=r$. Therefore if $r \geqslant n-m+1$, then there exists $s \in \mathbb{N}$ such that $w^{s}(-r) \leqslant n-m$ and $w^{s+1}(-r) \geqslant n-m+1$. In other words,

$$
w\{n-m, n-m+1\} \cap\left(I_{j} \sqcup-I_{j}\right) \neq \emptyset .
$$

Therefore

$$
w[n-m, n-m+1] \geqslant\left|\left\{j ; \max I_{j} \geqslant n-m+1\right\}\right| .
$$

Let $J=\left\{j ; \max I_{j} \geqslant n-m+1\right\}$. Note that there are exactly $m$ elements in $I_{1} \sqcup \ldots \sqcup I_{l}$ that are larger than or equal to $n-m+1$. We have $\sum_{j \in J}\left|I_{j}\right| \geqslant m$. As $\left|I_{k}\right|=a_{k}$ for all $k$ and $a_{1} \geqslant \cdots \geqslant a_{l}$, we have $a_{1}+\cdots+a_{|J|} \geqslant \sum_{j \in J}\left|I_{j}\right| \geqslant m$. In other words, $|J| \geqslant \min \left\{k ; a_{1}+\cdots+a_{k} \geqslant m\right\}$. The proposition is proved.

Proof of Proposition 3.5 for type $B / C$. For any partition $\alpha=\left(a_{1}, \ldots, a_{l}\right)$ of $n$, we define

$$
w_{\alpha}=\left(s_{\left[2, n+1-a_{1}\right]}^{-1} s_{[1, n]}\right)\left(s_{\left[2, n+1-a_{1}-a_{2}\right]}^{-1} s_{\left[1, n-a_{1}\right]}\right) \cdots\left(s_{\left[1, a_{l}\right]}\right) .
$$

By [He07, Lemma 7.15], $w_{\alpha}$ is a minimal length element in $\mathcal{C}_{\alpha}$.

If $\alpha \geqslant \beta$, then

$$
\alpha_{1} \geqslant \beta_{1}, \alpha_{1}+\alpha_{2} \geqslant \beta_{1}+\beta_{2}, \ldots
$$

Thus

$$
\begin{gathered}
s_{\left[2, n+1-\alpha_{1}\right]}^{-1} s_{[1, n]} \leqslant s_{\left[2, n+1-\beta_{1}\right]}^{-1} s_{[1, n]}, \\
s_{\left[2, n+1-\alpha_{1}-\alpha_{2}\right]}^{-1} s_{\left[1, n-\alpha_{1}\right]} \leqslant s_{\left[2, n+1-\beta_{1}-\beta_{2}\right]}^{-1} s_{\left[1, n-\beta_{1}\right]},
\end{gathered}
$$

So $w_{\alpha} \leqslant w_{\beta}$ and thus $\mathcal{C}_{\alpha} \preceq_{W} \mathcal{C}_{\beta}$.

On the other hand, if $\mathcal{C}_{\alpha} \preceq_{W} \mathcal{C}_{\beta}$, then there exists $w \in \mathcal{C}_{\alpha}$ with $w \leqslant w_{\beta}$. Let $m=\beta_{1}+\cdots+\beta_{k}$. By direct computation, we have $w_{\beta}[n-m, n-m+1]=k$. By Proposition 5.1, $w[n-m, n-m+1] \leqslant k$. By Proposition 5.1(2), $k \geqslant \min \left\{k^{\prime} ; \alpha_{1}+\right.$ $\left.\cdots+\alpha_{k^{\prime}} \geqslant m\right\}$. In other words, $\alpha_{1}+\cdots+\alpha_{k} \geqslant m=\beta_{1}+\cdots+\beta_{k}$. Therefore $\alpha \geqslant \beta$.

Proof of Proposition 3.5 for type $D$. Let $\delta$ be the permutation of $\{ \pm 1, \ldots, \pm n\}$ such that $\delta(1)=-1$ and $\delta(k)=k$ for $2 \leqslant k \leqslant n$. Then $W=W^{0} \sqcup W^{0} \delta$. For any partition $\alpha$, the corresponding conjugacy class $\mathcal{C}_{\alpha}$ is contained in $W^{0}$ if $\alpha_{1}^{*}$ is even and is contained in $W^{0} \delta$ if $\alpha_{1}^{*}$ is odd. 
For $0 \leqslant a<b \leqslant n$, define

$$
w_{a, b}= \begin{cases}s_{[3, n+1-b]}^{-1} s_{[1, n-a]}, & \text { if } b \leqslant n-1 \\ 1, & \text { otherwise }\end{cases}
$$

For any partition $\alpha=\left(a_{1}, \ldots, a_{l}\right)$ of $n$, we define

$$
w_{\alpha}^{\prime}= \begin{cases}w_{\left[0, \alpha_{1}\right]} w_{\left[\alpha_{1}, \alpha_{1}+\alpha_{2}\right]} \cdots w_{\sum_{1 \leqslant i \leqslant l-1} \alpha_{i}, n}, & \text { if } 2 \mid \alpha_{1}^{*} ; \\ w_{\left[0, \alpha_{1}\right]} w_{\left[\alpha_{1}, \alpha_{1}+\alpha_{2}\right]} \cdots w_{\sum_{1 \leqslant i \leqslant l-1} \alpha_{i}, n} \delta, & \text { if } 2 \nmid \alpha_{1}^{*} .\end{cases}
$$

By [He07, Lemma 7.19], $w_{\alpha}^{\prime}$ is a minimal length element in $\mathcal{C}_{\alpha}$.

Similar to the proof of the Proposition in type $B / C$, we have $w_{\alpha}^{\prime} \leqslant w_{\beta}^{\prime}$ if $\alpha \geqslant \beta$.

On the other hand, if $\mathcal{C}_{\alpha} \preceq_{W} \mathcal{C}_{\beta}$, then there exists $w \in \mathcal{C}_{\alpha}$ with $w \leq w_{\beta}^{\prime}$. Let $m=\beta_{1}+\cdots+\beta_{k}$. By direct computation, we have $w_{\beta}^{\prime}[n-m, n-m+1]=k$. By Proposition 5.1(3) $w[n-m, n-m+1] \leqslant k$. By Proposition 5.4, $k \geqslant \min \left\{k^{\prime} ; \alpha_{1}+\right.$ $\left.\cdots+\alpha_{k^{\prime}} \geqslant m\right\}$. In other words, $\alpha_{1}+\cdots+\alpha_{k} \geqslant m=\beta_{1}+\cdots+\beta_{k}$. Therefore $\alpha \geqslant \beta$.

5.2. Case 2: Type ${ }^{2} A_{n-1}$. Let $W=S_{n}$ be the group of permutations of $\{1,2, \cdots, n\}$. For $1 \leqslant i, j \leqslant n$, we define

$$
w\{i, j\}=\{-n \leqslant k \leqslant n ; k \leqslant i, w(k) \geqslant j\}, \quad w[i, j]=|w\{i, j\}| .
$$

Let $\delta=(1, n)(2, n-1) \cdots$ be the longest element of $W$. Then the conjugation action of $\delta$ on $W$ induces a bijection on the set of simple reflections and is a lengthpreserving automorphism. The map $W \rightarrow W: w \mapsto w \delta$ induces a bijection from the set of $\delta$-twisted conjugacy class of $W$ to the set of ordinary conjugacy classes of $W$. Since $\delta$ is the longest element of $W$, the map is order-reversing.

The $\delta$-twisted elliptic conjugacy classes of $W$ are parametrized by the partitions of $n$ with odd parts. Let $\alpha=\left(\alpha_{1}, \alpha_{2}, \ldots, \alpha_{l}\right)$ be a partition of $n$ with odd parts, i.e. $\alpha_{1} \geqslant \alpha_{2} \geqslant \cdots \geqslant \alpha_{l}>0$ are odd positive integers and $\alpha_{1}+\cdots+\alpha_{l}=n$. Let $\mathcal{C}_{\alpha}$ be the corresponding $\delta$-twisted elliptic conjugacy class in $W$. This is the conjugacy class of $W$ consisting of permutations $w$ satisfying the following conditions:

(1) There exists a decomposition $\{1,2, \ldots, n\}=I_{1} \sqcup I_{2} \sqcup \ldots \sqcup I_{l}$;

(2) $\left|I_{j}\right|=\alpha_{j}$ for all $j$;

(3) The orbits of $w \delta$ on $\{1,2, \ldots, n\}$ are $I_{j}$ for $1 \leqslant j \leqslant l$.

We have the following useful inequalities for the elements in $\mathcal{C}_{\alpha}$.

Proposition 5.5. Let $w \in \mathcal{C}_{\alpha}$ and $1 \leqslant m \leqslant n-1$, we have $w \delta\left[\left\lceil\frac{m}{2}\right\rceil, n-\left\lfloor\frac{m}{2}\right\rfloor+1\right]+w \delta\left[n-\left\lfloor\frac{m}{2}\right\rfloor,\left\lceil\frac{m}{2}\right\rceil+1\right] \leqslant n-\min \left\{k ; \alpha_{1}+\cdots+\alpha_{k} \geqslant m\right\}$.

Proof. Let $I=w \delta\left\{\left\lceil\frac{m}{2}\right\rceil, n-\left\lfloor\frac{m}{2}\right\rfloor+1\right\}$ and $I^{\prime}=w \delta\left\{n-\left\lfloor\frac{m}{2}\right\rfloor,\left\lceil\frac{m}{2}\right\rceil+1\right\}$. If $k \in I$, then $k \leqslant\left\lceil\frac{m}{2}\right\rceil<\left\lceil\frac{m}{2}\right\rceil+1$ and thus $(w \delta)^{-1}(k) \notin I^{\prime}$. In other words,

$$
I \cap(w \delta)\left(I^{\prime}\right)=\emptyset \text {. }
$$

Similarly,

$$
I \cap(w \delta)^{-1}\left(I^{\prime}\right)=\emptyset
$$


In particular, for any $w \delta$-orbit $I_{j}$, we have

$$
\left|\left(I_{j} \cap I\right)\right|+\left|\left(I_{j} \cap I^{\prime}\right)\right|=\left|\left(I_{j} \cap I\right)\right|+\left|\left(I_{j} \cap(w \delta)\left(I^{\prime}\right)\right)\right| \leqslant\left|I_{j}\right|
$$

We claim that

(b) If $\left|\left(I_{j} \cap I\right)\right|+\left|\left(I_{j} \cap I^{\prime}\right)\right|=\left|I_{j}\right|$, then $I_{j} \subset\left\{\left\lceil\frac{m}{2}\right\rceil+1,\left\lceil\frac{m}{2}\right\rceil+2, \ldots, n-\left\lfloor\frac{m}{2}\right\rfloor\right\}$. Note that

$$
\left.\left|I_{j} \cap I\right|+\left|I_{j} \cap w \delta\left(I^{\prime}\right)\right|\right)=\left|I_{j} \cap I\right|+\left|I_{j} \cap(w \delta)^{-1}\left(I^{\prime}\right)\right|=\left|I_{j}\right| .
$$

Since $I \cap(w \delta)\left(I^{\prime}\right)=I \cap(w \delta)^{-1}\left(I^{\prime}\right)=\emptyset$, we have

$$
\left(I_{j} \cap I\right) \sqcup\left(I_{j} \cap(w \delta)\left(I^{\prime}\right)\right)=\left(I_{j} \cap I\right) \sqcup\left(I_{j} \cap(w \delta)^{-1}\left(I^{\prime}\right)\right)=I_{j} .
$$

Thus

$$
(w \delta)\left(I_{j} \cap I^{\prime}\right)=I_{j} \cap(w \delta)\left(I^{\prime}\right)=I_{j} \cap(w \delta)^{-1}\left(I^{\prime}\right)=(w \delta)^{-1}\left(I_{j} \cap I^{\prime}\right) .
$$

In other words, $I_{j} \cap I^{\prime}$ is a subset of $I_{j}$ that is stable under the action of $(w \delta)^{2}$. Since the order of the action of $w \delta$ on $I_{j}$ equals to $\left|I_{j}\right|$, which is an odd integer. Hence $I_{j} \cap I^{\prime}$ is a $w \delta$-stable subset of $I_{j}$. As $w \delta$ acts transitively on $I_{j}$, we have $I_{j} \cap I^{\prime}=\emptyset$ or $I_{j}$. Hence $I_{j} \subset I$ or $I_{j} \subset I^{\prime}$. However, as $\left\lceil\frac{m}{2}\right\rceil<n-\left\lfloor\frac{m}{2}\right\rfloor+1$, if $k \in I$, then $(w \delta)(k) \notin I$. Thus $I_{j} \subset I^{\prime}$. In other words, for any $k \in I_{j}, k \geqslant\left\lceil\frac{m}{2}\right\rceil+1$ and $k \leqslant n-\left\lfloor\frac{m}{2}\right\rfloor$.

(b) is proved.

Let $J=\left\{j ; I_{j} \not \subset\left\{\left\lceil\frac{m}{2}\right\rceil+1,\left\lceil\frac{m}{2}\right\rceil+2, \ldots, n-\left\lfloor\frac{m}{2}\right\rfloor\right\}\right\}$. By (a) and (b), we have that

$$
|J|+\left|J^{\prime}\right|=\sum_{j}\left(\left|I_{j} \cap J\right|+\left|I_{j} \cap J^{\prime}\right|\right) \leqslant \sum_{j}\left|I_{j}\right|-|J|=n-|J| .
$$

Note that there are exactly $m$ elements outside $\left\{\left\lceil\frac{m}{2}\right\rceil+1,\left\lceil\frac{m}{2}\right\rceil+2, \ldots, n-\left\lfloor\frac{m}{2}\right\rfloor\right\}$. We have $\sum_{j \in J}\left|I_{j}\right| \geqslant m$. As $\left|I_{k}\right|=\alpha_{k}$ for all $k$ and $\alpha_{1} \geqslant \cdots \geqslant \alpha_{l}$, we have $\alpha_{1}+\cdots+\alpha_{|J|} \geqslant \sum_{j \in J}\left|I_{j}\right| \geqslant m$. In other words, $|J| \geqslant \min \left\{k ; \alpha_{1}+\cdots+\alpha_{k} \geqslant m\right\}$. The proposition is proved.

Now we prove the following result.

Proof of Proposition 3.5 for type ${ }^{2} A_{n-1}$. The strategy is similar to the proof of the Proposition in types B/C.

For any partition $\alpha=\left(a_{1}, \ldots, a_{l}\right)$ of $n$ consisting of only odd parts, we define

$$
w_{\alpha}^{\prime \prime}=s_{\left[1, n+1-\frac{a_{1}+1}{2}\right]}^{-1} s_{\left[\frac{a_{1}+1}{2}+1, n+2-\frac{a_{1}+1}{2}-\frac{a_{2}+1}{2}\right]}^{-1} \cdots s_{\left[\sum_{1 \leqslant i \leqslant l-1} \frac{a_{i}+1}{2}+1, n+l-\sum_{1 \leqslant i \leqslant l} \frac{a_{i}+1}{2}\right]} \delta .
$$

By [He07, Lemma 7.13], $w_{\alpha}^{\prime \prime}$ is a minimal length element in $\mathcal{C}_{\alpha}$.

If $\alpha \geqslant \beta$, then $\alpha_{1} \geqslant \beta_{1}, \alpha_{1}+\alpha_{2} \geqslant \beta_{1}+\beta_{2}, \ldots$. By the explicit formula, $w_{\alpha}^{\prime \prime} \leqslant w_{\beta}^{\prime \prime}$ and thus $\mathcal{C}_{\alpha} \preceq_{W} \mathcal{C}_{\beta}$.

On the other hand, if $\mathcal{C}_{\alpha} \preceq_{W} \mathcal{C}_{\beta}$, then there exists $w \in \mathcal{C}_{\alpha}$ with $w \leqslant w_{\beta}^{\prime \prime}$. Hence $w w_{0} \geqslant w_{\beta}^{\prime \prime} w_{0}$, where $w_{0}=(1 n)(2, n-1) \cdots$. Let $m=\beta_{1}+\cdots+\beta_{k}$. By direct computation, we have

$$
w_{\beta} w_{0}\left[\left\lceil\frac{m}{2}\right\rceil, n-\left\lfloor\frac{m}{2}\right\rfloor+1\right]+w_{\beta} w_{0}\left[n-\left\lfloor\frac{m}{2}\right\rfloor,\left\lceil\frac{m}{2}\right\rceil+1\right]=n-k .
$$

By Proposition 5.1(1),

$$
w w_{0}\left[\left\lceil\frac{m}{2}\right\rceil, n-\left\lfloor\frac{m}{2}\right\rfloor+1\right]+w w_{0}\left[n-\left\lfloor\frac{m}{2}\right\rfloor,\left\lceil\frac{m}{2}\right\rceil+1\right] \geqslant n-k .
$$


By Proposition 5.5, $k \geqslant \min \left\{k^{\prime} ; \alpha_{1}+\cdots+\alpha_{k^{\prime}} \geqslant m\right\}$. In other words, $\alpha_{1}+\cdots+\alpha_{k} \geqslant$ $m=\beta_{1}+\cdots+\beta_{k}$. Therefore $\alpha \geqslant \beta$.

\section{Exceptional Groups}

We prove Theorem 1.1 for exceptional groups by computer computation, using the Atlas of Lie Groups and Representations software [A].

Suppose $G$ is a simple, untwisted exceptional group. We first list representatives of the elliptic conjugacy classes in the Weyl group, and we choose these representatives to be of minimal length. We use the representatives of [GP00, Appendix $\mathrm{B}]$, and we name the classes according to these tables. We explicitly compute the order relation on these classes.

We label the unipotent classes in characteristic 0 as in [Sp82, Section IV.2]. Using the explicit description of Lusztig's map $\Phi$ [Lu11, Section 4.3], and the closure relations for unipotent orbits of [Sp82, Section IV.2], we compute the Hasse diagram for the image of $\Phi$.

In the twisted cases ${ }^{3} D_{4}$ and ${ }^{2} E_{6}$ we label the elliptic conjugacy classes as in [Lu12b]. This uses the label of the ordinary conjugacy class, from the CHEVIE software in type ${ }^{3} D_{4}$, and from [Ca72] in ${ }^{2} E_{6}$, and in both cases we also use ${ }^{!}$to indicate the twisted class is elliptic. The labelling of unipotent classes classes is from [Sp82], page $148\left({ }^{3} D_{4}\right)$ and page $250\left({ }^{2} E_{6}\right)$, followed by (in brackets) that of [Ma93] and [Ma93a]. Lusztig defines the map $\Phi$ using the latter notation.

In each case we give the bijection between elliptic conjugacy classes and unipotent orbits and the corresponding Hasse diagram.

We explain the notation using the example of $G_{2}$.

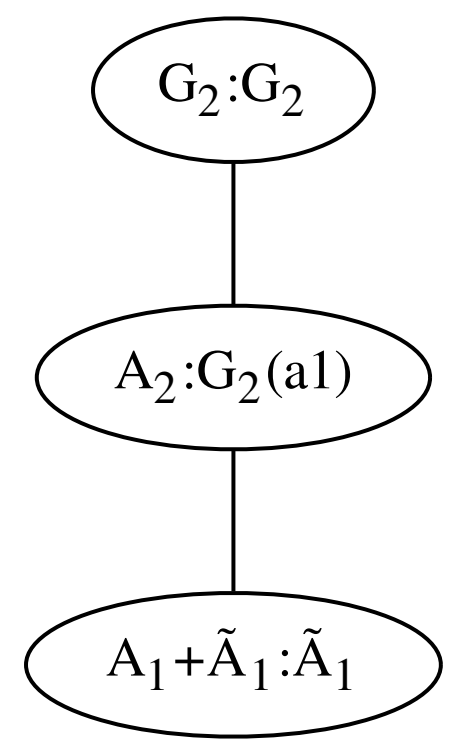

The left-hand entries $G_{2}, A_{2}$ and $A_{1}+\widetilde{A_{1}}$ are the elliptic conjugacy classes of the Weyl group, of orders 6 (the Coxeter element of $G_{2}$ ), 3 (the Coxeter element 
of $A_{2}$ ) and 2 (the Coxeter element of $A_{1}+\widetilde{A_{1}}$ ), respectively. The order relation is:

$$
G_{2} \preceq_{W} A_{2} \preceq_{W} A_{1}+\widetilde{A_{1}} .
$$

The entry following the colon is a unipotent class in $G_{2}(\mathbb{C})$, labeled as in [Sp82]; the image of the corresponding elliptic conjugacy class under the Lusztig map. These are $G_{2}, G_{2}\left(a_{1}\right)$ and $\widetilde{A_{1}}$, of dimension 12 (the regular orbit), 10 (the subregular orbit) and 6 respectively, of which the first two are distinguished. Here the order is the opposite one.

$$
\widetilde{A_{1}} \preceq_{u} G_{2}\left(a_{1}\right) \preceq_{u} G_{2} .
$$

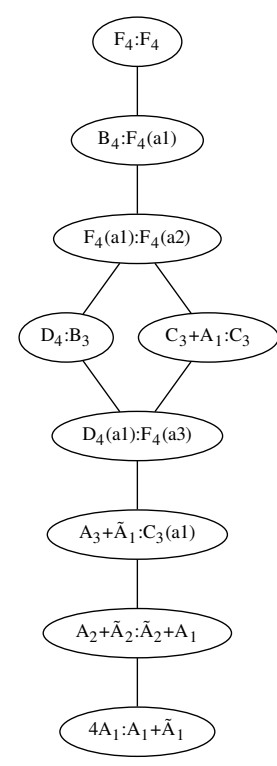

Type $F_{4}$

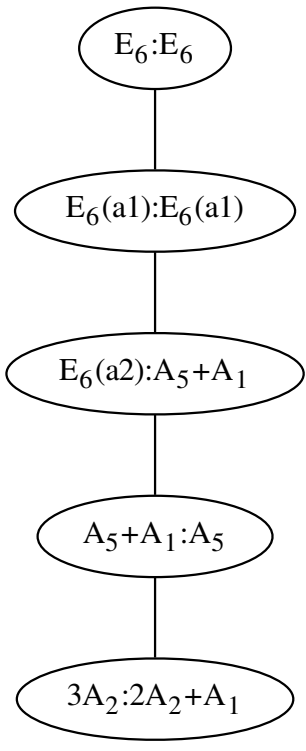

Type $E_{6}$ 


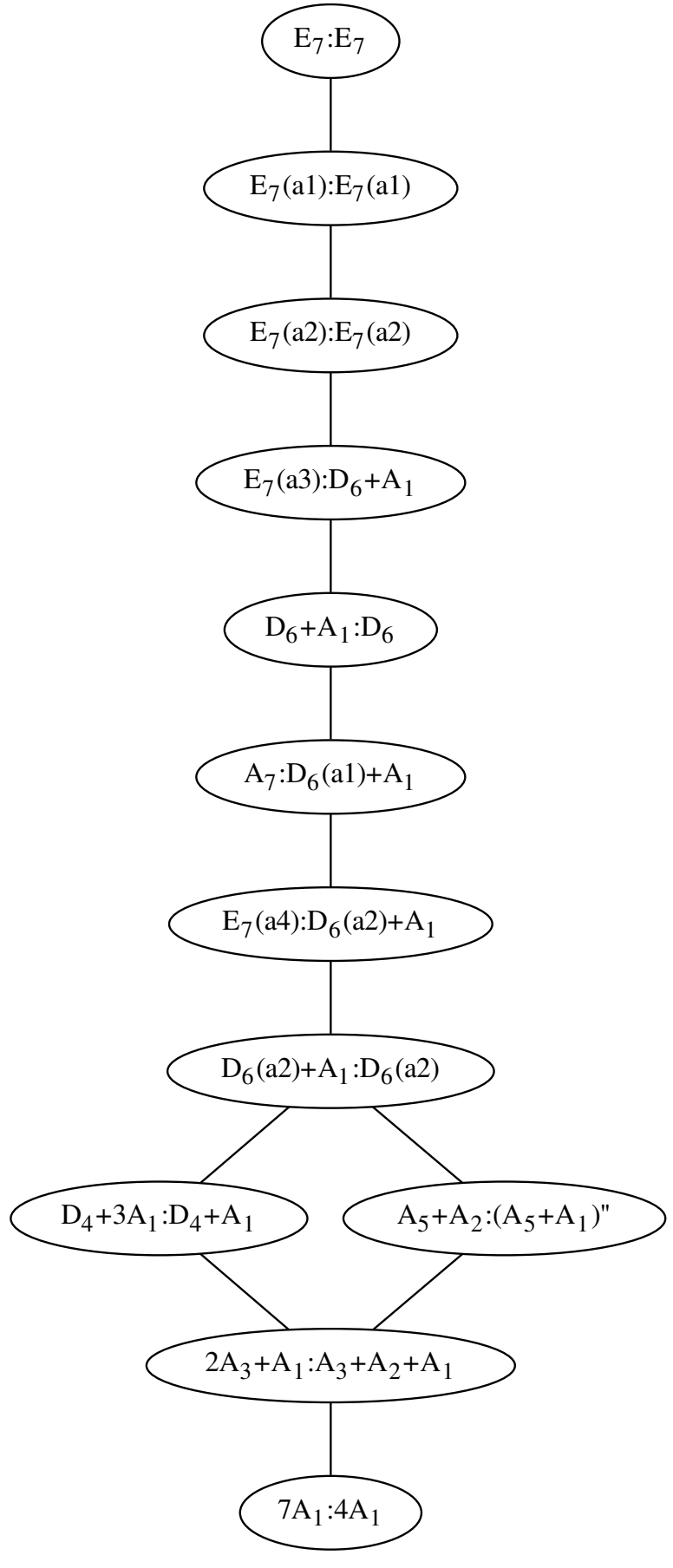

Type $E_{7}$

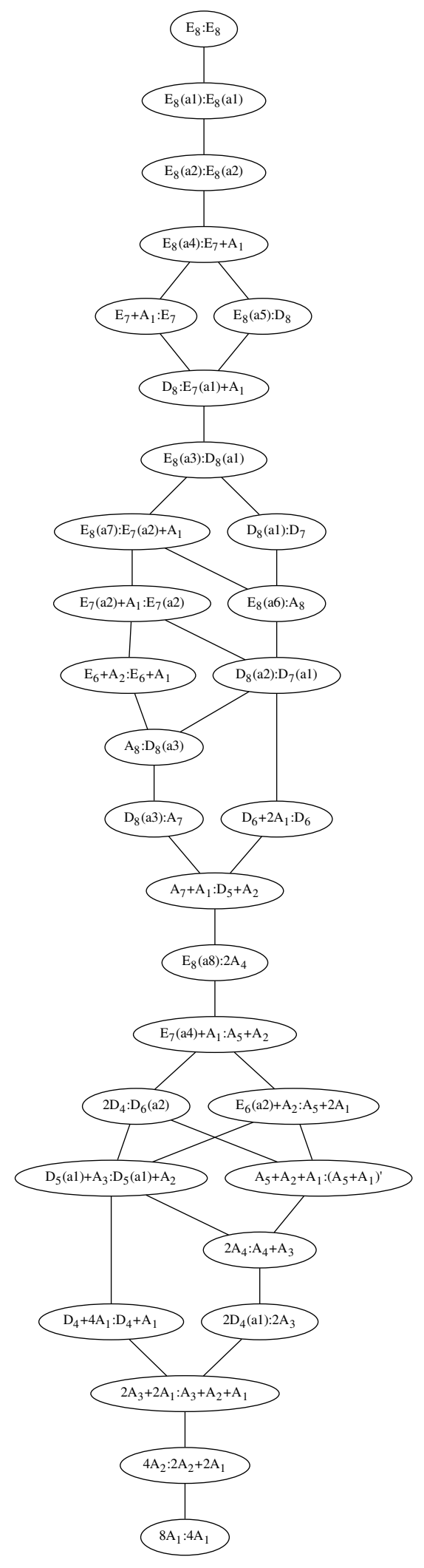

Type $E_{8}$ 

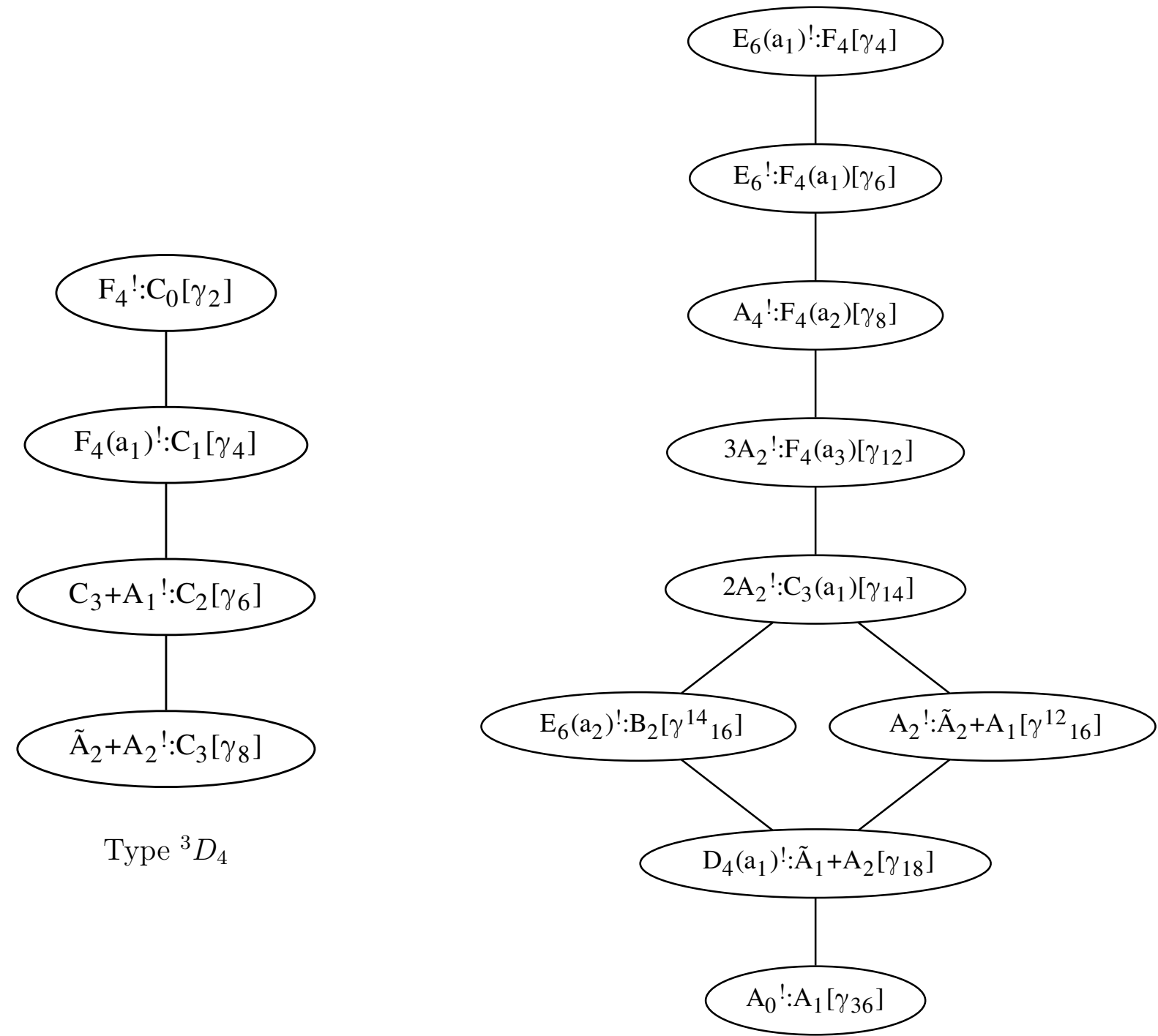

Type ${ }^{2} E_{6}$ 


\section{REFERENCES}

[A] Atlas of Lie Groups and Representations, www.liegroups.org, 2019

[BB05] F. Brenti and A. Björner, Combinatorics of Coxeter groups, Graduate Texts in Mathematics, vol. 231, Springer, New York (2005)

[Ca72] R. Carter, Conjugacy Classes in the Weyl Group, Compositio Math. 25 (1972), 1-59.

[CM93] D. Collingwood and M. McGovern, Nilpotent orbits in semisimple Lie algebras, Van Nostrand Reinhold Mathematics Series, Van Nostrand Reinhold Co., New York (1993)

[DHM13] O. Dudas, X. He and J. Michel, Private communication, 2013.

[DL76] P. Deligne and G. Lusztig, Representations of reductive groups over finite fields, Ann. of Math. (2) 103 (1976), no. 1, 103-161.

[DM15] O. Dudas and G. Malle, Decomposition matrices for low-rank unitary groups, Proc. Lond. Math. Soc. (3) 110 (2015), no. 6, 1517-1557.

[GP00] Meinolf Geck and Götz Pfeiffer, Characters of finite Coxeter groups and IwahoriHecke algebras, London Mathematical Society Monographs. New Series, vol. 21, The Clarendon Press, Oxford University Press, New York, 2000.

[Ha15] P. Hamacher, The geometry of Newton strata in the reduction modulo $p$ of Shimura varieties of PEL type, Duke Math. J. 164 (2015), no. 15, 2809-2895.

[HV11] U. Hartl and E. Viehmann, The Newton stratification on deformations of local Gshtukas, J. Reine Angew. Math. 656 (2011), 87-129.

[He07] X. He, Minimal length elements in some double cosets of Coxeter groups, Adv. Math. 215 (2007), 469-503.

[He14] X. He, Geometric and homological properties of affine Deligne-Lusztig varieties, Ann. of Math. (2) 179 (2014), 367-404.

[He16a] X. He, Hecke algebras and p-adic groups, Current developments in mathematics 2015, 73-135, Int. Press, Somerville, MA, 2016.

[He16b] X. He, Kottwitz-Rapoport conjecture on unions of affine Deligne-Lusztig varieties, Ann. Sci. Ècole Norm. Sup. 49 (2016), 1125-1141.

[HN14] X. He and S. Nie, Minimal length elements of extended affine Weyl group, Compos. Math. 150 (2014), 1903-1927.

[JO00] A. J. de Jong and F. Oort, Purity of the stratification by Newton polygons, J. Amer. Math. Soc. 13 (2000), 209-241.

[Lu03] G. Lusztig, Hecke algebras with unequal parameters, CRM Monographs Ser. 18, Amer. Math. Soc., Providence, RI, 2003; enlarged and updated version at arxiv.org/0208154.

[Lu03] G. Lusztig, Character sheaves on disconnected groups I, Represent.Theory 7 (2003), 374-403.

[Lu11] G. Lusztig, From conjugacy classes in the Weyl group to unipotent classes, Represent. Theory 15 (2011), 494-530.

[Lu12a] G. Lusztig, From conjugacy classes in the Weyl group to unipotent classes, II, Represent. Theory 16 (2012), 189-211.

[Lu12b] G. Lusztig, From conjugacy classes in the Weyl group to unipotent classes, III, Represent. Theory 16 (2012), 450-488.

[Ma93] G. Malle, Generalized Deligne-Lusztig characters, J.Algebra 159 (1993), 64-97.

[Ma93a] G. Malle, Green functions for groups of type E 6 and F 4 in characteristic 2, Commun.Algebra 21 (1993), 747-798.

[Sp82] N. Spaltenstein, Classes unipotents et sous-groupes de Borel, Lecture Notes in Math. 946, Springer, 1982.

[Vi13] E. Viehmann, Newton strata in the loop group of a reductive group, Amer. J. Math. 135 (2013), no. 2, 499-518.

[Wa63] G. E. Wall, On the conjugacy classes in the unitary, symplectic and orthogonal groups, J. Austral. Math. Soc., 3 (1963), 1-63. 
The Institute of Mathematical Sciences and Department of Mathematics, The Chinese University of Hong Kong, Shatin, N.T., Hong Kong, xuhuahe@gmail.com

Institute of Mathematics, Academy of Mathematics and Systems Science, Chinese Academy of Sciences, 100190, Beijing, China, niesian@amss.ac.CN 